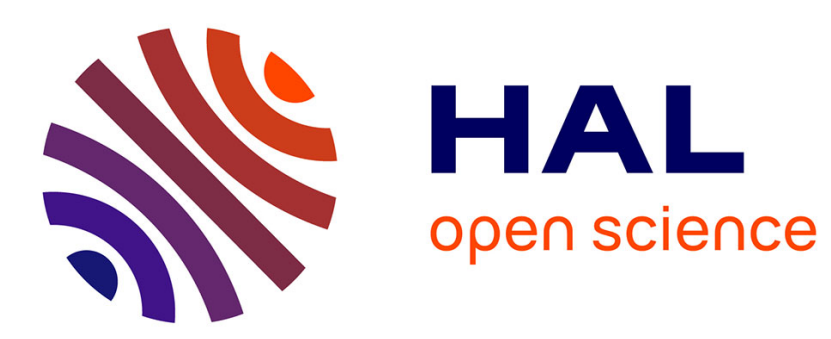

\title{
L'évolution de l'écriture épigraphique en France au Moyen Âge et ses enjeux historiques
}

\author{
Vincent Debiais, Robert Favreau, Cécile Treffort
}

\section{To cite this version:}

Vincent Debiais, Robert Favreau, Cécile Treffort. L'évolution de l'écriture épigraphique en France au Moyen Âge et ses enjeux historiques. Bibliotheque- Ecole des Chartes, 2008, 165 (1), pp.101-137. 10.3406/bec.2007.463492 . halshs-00343581

\section{HAL Id: halshs-00343581 https://shs.hal.science/halshs-00343581}

Submitted on 2 Dec 2008

HAL is a multi-disciplinary open access archive for the deposit and dissemination of scientific research documents, whether they are published or not. The documents may come from teaching and research institutions in France or abroad, or from public or private research centers.
L'archive ouverte pluridisciplinaire HAL, est destinée au dépôt et à la diffusion de documents scientifiques de niveau recherche, publiés ou non, émanant des établissements d'enseignement et de recherche français ou étrangers, des laboratoires publics ou privés. 


\title{
L'ÉVOLUTION DE L'ÉCRITURE ÉPIGRAPHIQUE EN FRANCE AU MOYEN ÂGE ET SES ENJEUX HISTORIQUES
}

\author{
par \\ Vincent DEBIAIS, Robert FAVREAU \\ et CÉcIle TREFFORT
}

Longtemps caractérisée par la nature de son support, l'inscription médiévale est aujourd'hui définie par sa fonction, celle de transmettre des informations de toute nature pour le temps le plus long et au public le plus large possibles ${ }^{1}$. Destinés à répondre à un désir de publicité, la technique de réalisation, la dimension ou encore l'emplacement des inscriptions sont donc des éléments constitutifs de cette source historique particulière qui associe intimement le verbe à la matière. La mise en page, la composition du texte et les choix graphiques relèvent de cette même logique : attirer l'attention du passant pour le transformer en lecteur et lui permettre de s'approprier non seulement le contenu du discours, mais également son contexte : caractère plus ou moins officiel, plus ou moins dynamique, plus ou moins savant...

Les enjeux d'une étude de l'écriture épigraphique au Moyen Âge dépassent donc la vocation instrumentale d'une analyse visant essentiellement à la datation du document dans une perspective positiviste. Les caractéristiques paléographiques des inscriptions, montrant d'importantes variations dans le temps comme dans l'espace, dessinent ainsi des ensembles culturels sur lesquels l'historien doit s'interroger. L'écriture accompagne, voire se mêle intimement aux décors et aux images, créant un discours graphique mixte qui commence également à retenir l'attention des médiévistes. Enfin, en tant que manifestation de l'écrit, l'inscription est en rapport étroit avec la culture littéraire du temps, son impact restant tributaire de la nature et de la profondeur de

1. Panorama général dans Robert Favreau, Les inscriptions médiévales, Turnhout, 1979 (Typologie des sources du Moyen Âge occidental, 35) et id., Épigraphie médiévale, Turnhout, 1997 (L’atelier du médiéviste, 5).

Vincent Debiais, ingénieur de recherche ; Robert Favreau, professeur émérite ; Cécile Treffort, professeur : Université de Poitiers, Centre d'études supérieures de civilisation médiévale, 24 rue de la Chaîne, BP 603, 86022 Poitiers Cedex. <vincent.debiais@univ-poitiers.fr> <cecile.treffort@ univ-poitiers.fr> 
l'alphabétisation de la société médiévale qui la reçoit. Entre tradition et innovation, les grands changements de l'écriture épigraphique peuvent aujourd'hui devenir un véritable objet d'histoire.

Les synthèses en ce domaine restent rares; tout au plus peut-on signaler, pour l'Allemagne, le manuel de Rudolf Kloos et, pour la France, l'article déjà ancien mais non encore remplacé de Paul Deschamps, ainsi que, pour l'Antiquité tardive et le haut Moyen Âge, celui d'Edmond Le Blant ${ }^{2}$. Plus généralement, le riche dossier consacré en 1981 aux rapports entre épigraphie et paléographie par la revue Scrittura e civiltà ${ }^{3}$; le panorama dressé en 1997 pour la France par R. Favreau dans son Épigraphie médiévale, où l'on trouvera les références de tous les articles alors publiés mais limités à une période ou à une région ; enfin le tout récent manuel de Walter Koch ${ }^{4}$.

Cet état d'avancement de la recherche est tributaire de l'émergence récente de la discipline épigraphique dans les études médiévales et du caractère incomplet des corpus de référence. Sans vouloir fournir ici un tableau exhaustif des publications en matière d'inscriptions médiévales, brossé de manière précise en d'autres lieux, on peut souligner que, à part la Suisse et son Corpus inscriptionum Medii Aevi Helvetiae pour les inscriptions antérieures à 1300, aucune des grandes collections européennes, du Corpus des inscriptions de la France médiévale ${ }^{5}$ aux Deutschen Inschriften, en passant par les Inscriptiones Medii Aevi Italiae ou d'autres recueils encore ${ }^{6}$, n'est actuellement achevée. Or, non seulement la documentation épigraphique est extrêmement dispersée mais elle doit faire l'objet d'une analyse critique fondée sur l'observation directe pour caractériser avec rigueur son écriture et pour dater avec précision sa réalisation matérielle. Cet article vise donc moins à proposer une véritable synthèse, impossible dans l'état actuel de la recherche même à l'échelle d'un seul pays comme la France, qu'à clarifier quelques préalables méthodologiques, à dessiner les grandes lignes de l'évolution paléographique telle qu'on peut l'appréhender aujourd'hui et à en mettre en lumière les enjeux historiques du début à la fin de la période médiévale.

2. Rudolf M. Kloos, Einführung in die Epigraphik des Mittelalters und der frühen Neuzeit, Darmstadt, 1980 (l'évolution morphologique est intéressante à comparer avec celle des inscriptions françaises, dont Kloos note qu'elle est plus précoce) ; Paul Deschamps, «Étude sur la paléographie des inscriptions lapidaires de la fin de l'époque mérovingienne aux dernières années du XII siècle ", dans Bulletin monumental, t. 88, 1929, p. 5-81 ; Edmond Le Blant, « Paléographie des inscriptions latines du III $^{\mathrm{e}}$ à la fin du viI ${ }^{\mathrm{e}}$ siècle ", dans Revue archéologique, $3^{\mathrm{e}}$ sér., t. 29, 1896, p. 177-197 et 345-355 ; t. 30, 1897, p. 30-40 et 171-184 ; t. 31, 1897, p. 172-184.

3. "Epigrafia e paleografia : inchiesta sui rapporti fra due discipline ", dans Scrittura e civiltà, t. 5, 1981, p. 265-312.

4. R. Favreau, Épigraphie..., chap. 3 («Introduction à une paléographie des inscriptions »), p. 57-89 ; Walter Koch, Inschriftenpaläographie des abendländischen Mittelalters und der früheren Neuzeit, t. I : Früh- und Hochmittelalter, Vienne-Munich, 2007.

5. Corpus des inscriptions de la France médiévale, éd. R. Favreau et Jean Michaud, collab. Bernadette Mora pour les t. V à XXI et Giuseppe Di Spirito pour le t. XXII, Poitiers [puis] Paris, depuis 1974, 22 vol. parus [désormais abrégé : CIFM].

6. L’ensemble des grands corpus est détaillé dans R. Favreau, Épigraphie..., p. 10-23. 
Si l'on s'en tient au territoire français, l'étude repose sur une documentation de nature et de valeur diverses suivant les périodes et les régions. Pour le haut Moyen Âge, les recueils d'E. Le Blant ${ }^{7}$ restent en usage pour une grande partie du territoire, celle qui n'a pas encore été couverte par le Recueil des inscriptions chrétiennes de la Gaule antérieures à la réforme carolingienne ${ }^{8}$ dont seuls trois volumes, consacrés respectivement aux provinces de Trèves, Vienne et Bourges, sont parus. On peut d'autant plus le regretter que la qualité de l'étude paléographique, accompagnée de tableaux synthétiques et de reproductions nombreuses, y est remarquable. Pour la période postérieure, entre le $\mathrm{IX}^{\mathrm{e}}$ et le XIII ${ }^{\mathrm{e}}$ siècle, on dispose du Corpus des inscriptions de la France médiévale ${ }^{9}$, entreprise nationale centralisée à Poitiers. Les vingt-deux volumes parus depuis la création de l'équipe en 1969, ainsi que ceux actuellement en préparation, couvrent plus des deux tiers sud et ouest de la France. La documentation photographique réunie dans les locaux du Centre d'études supérieures de civilisation médiévale (CESCM), riche de plus de six mille clichés et accompagnée d'un fichier paléographique manuel, fournit une base de travail solide. Elle est malheureusement encore incomplète d'un point de vue géographique puisque le nord et l'est de la France ne seront traités que dans les prochaines années. En outre, la publication actuelle ne couvrant que les $\mathrm{IX}^{\mathrm{e}}$-xIII ${ }^{\mathrm{e}}$ siècles, les inscriptions de la fin du Moyen Âge n'y figurent pas. Même si ces dernières sont systématiquement répertoriées dans un fichier manuel en cours de numérisation, elles ne sont concernées que de manière marginale par les missions photographiques du Corpus. Pour l'étude paléographique des XIV et $\mathrm{Xv}^{\mathrm{e}}$ siècles, force est donc de recourir aux recueils régionaux, souvent anciens, où relevés et dessins sont d'une fiabilité variable, ou à des études ponctuelles dont la dispersion bibliographique rend difficile le repérage. Cette hétérogénéité à la fois chronologique et géographique de la documentation actuellement disponible ne peut qu'inciter le chercheur à la prudence.

Un deuxième point méthodologique doit être souligné : la prégnance des inscriptions lapidaires dans l'élaboration des tableaux paléographiques, et en particulier de celui proposé par P. Deschamps ${ }^{10}$. Or, la diversité des supports et des techniques de réalisation oblige à s'interroger sur la validité d'un tel référent. La percussion d'un outil destiné, par l'enlèvement de la matière, à créer un relief, donc l'ombre qui crée la lettre, s'apparente à la sculpture et relève d'un geste bien différent de celui qui, par le mouvement fluide de la

7. E. Le Blant, Les inscriptions chrétiennes de la Gaule antérieures au VIII siècle, t. I : Provinces gallicanes, t. II : Les sept provinces, Paris, 1856-1865, 2 vol. (rééd. Hildesheim-ZurichNew York, 1999), et Nouveau recueil des inscriptions chrétiennes de la Gaule antérieures au VIII siècle, Paris, 1892 (Collection de documents inédits sur l'histoire de France).

8. Recueil des inscriptions chrétiennes de la Gaule antérieures à la Renaissance carolingienne (RICG), Paris, depuis 1975 : t. I, Nancy Gauthier, Première Belgique, 1975 ; t. XV-1, Françoise Descombes, Viennoise du Nord, 1985 ; t. VIII, Françoise Prévot, Aquitaine première, 1997.

9. Voir note 5. Pour des informations générales sur le fonctionnement de l'équipe, on peut consulter le site internet du CESCM à l'adresse suivante : www.mshs.univ-poitiers.fr/cescm/.

10. P. Deschamps, «Paléographie des inscriptions... », p. 63-82. 
main, aboutit à un tracé pictural peut-être plus proche des traditions manuscrites. Le procédé utilisé peut ainsi avoir des conséquences importantes sur la graphie du texte : sculpture sur pierre ou sur bois, peinture murale, mosaïque, vitraux, tapisseries ou broderies, métal repoussé, fondu ou niellé exercent une contrainte technique importante que l'on n'a pas toujours assez pris en compte.

L'application à toutes les inscriptions médiévales, quel que soit leur support, d'une analyse fondée sur des témoignages exclusivement lapidaires n'est de fait pas sans danger, même si elle reste parfois le seul recours possible. En effet, s'il est indispensable de s'appuyer sur des documents portant leur date (datés ou datables, selon la distinction établie par les paléographes), on manque cruellement de référent chronologique fiable pour certains supports, en particulier pour les peintures murales. Il ne faut pas exclure non plus que les inscriptions datées, en particulier dans la première moitié du Moyen Âge où elles sont plus rares, puissent être plus soignées que les autres et donc médiocrement représentatives de la production contemporaine.

Il convient enfin de s'interroger sur la notion même d'écriture dans le cadre des inscriptions médiévales et, surtout, sur les critères pertinents pour analyser son évolution au cours des siècles. À la fois acte physique, production intellectuelle et résultat, l'écriture varie ainsi non seulement par la forme des lettres (la morphologie) mais également par l'agencement de ces dernières dans l'espace et par l'adjonction d'autres signes graphiques (la ponctuation) permettant une correspondance du texte avec un discours oral, l'acte d'écrire étant étroitement lié à celui de lire. Si l'on peut parler sans conteste d'évolution de l'écriture épigraphique, celle-ci n'est ni linéaire ni uniforme : entre la transformation lente de certaines lettres et la création de formes graphiques nouvelles, on trouve également des retours à des modèles anciens, qualifiés de « renaissances ", voire des systèmes mixtes. Si plusieurs périodes sont marquées par une nette uniformisation de l'écriture, certaines variations peuvent néanmoins être induites par la coexistence de plusieurs niveaux d'exécution dans la production épigraphique.

Si une étude de l'évolution de l'écriture épigraphique au Moyen Âge reste possible, elle doit donc nécessairement reposer sur ces précautions méthodologiques : en nuançant les observations retenues, elles ne les rendront que plus fiables.

\section{Le haut Moyen Âge.}

Parmi les grandes phases chronologiques qui structurent, d'un point de vue épigraphique, la période médiévale, le haut Moyen Âge occupe une place à part, non seulement parce qu'il opère la transition, lente et parfois chaotique, à partir des traditions antiques, mais également parce que l'inscription doit se comprendre alors dans le cadre d'une société faiblement alphabétisée. Même si cette situation n'empêche pas l'écrit de garder une vraie force évocatrice, voire 
magique, les rapports entre paléographie et lecture effective se posent en des termes autres que dans les siècles suivants. La hiérarchisation des niveaux d'écriture y est sans doute particulièrement significative, notamment au moment du tournant carolingien, où l'écriture dans son ensemble fait l'objet d'une attention toute particulière.

Certains éléments sont communs à l'ensemble de la période qui s'étend de l'époque mérovingienne à l'an mille, en particulier l'usage de la scriptio continua, écriture continue sans séparation des mots ni ponctuation. La compacité de ces textes est renforcée par la disparition presque complète d'éléments adventices comme les feuilles de fin de ligne employées dans l'Antiquité tardive, ou de compléments iconographiques comme les portraits des défunts, absents des épitaphes jusqu'à leur réapparition au $\mathrm{XI}^{\mathrm{e}}$ siècle. Si l'on excepte les didascalies, c'est-à-dire les légendes des programmes iconographiques, les inscriptions du haut Moyen Âge se distinguent très nettement des images. Principalement composé d'inscriptions funéraires sur support lapidaire ${ }^{11}$, le corpus des inscriptions mérovingiennes et carolingiennes se présente comme un ensemble de pierres quadrangulaires, de dimensions, matières et mises en œuvre diverses, mais couvertes de textes mis en page comme sur un parchemin. Le terme de pagina qu'emploie au vi ${ }^{\mathrm{e}}$ siècle Sidoine Apollinaire pour désigner la plaque de marbre sur laquelle il souhaite faire graver l'épitaphe de son grand-père ${ }^{12}$ est à ce titre très significatif.

La scriptio continua, par son unité sinon sa régularité graphique et la cohérence des associations de lettres, se fait véritablement imago vocis, image de la parole, selon la définition des grammairiens antiques et médiévaux ${ }^{13}$. Elle n'est toutefois accessible qu'à des lecteurs savants, clercs pour la plupart, connaissant bien le latin et familiers des textes qu'ils ont sous les yeux. Ils pouvaient vraisemblablement lire sans difficulté une épitaphe où étaient inscrits le nom, la date du décès, une formule de prière, même s’il n'y avait pas de séparation entre les mots. La difficulté de lecture intervient quand le latin se fait langue savante ; on en a la démonstration dans les textes des îles Britanniques et de la Bretagne, où la différence entre latin et langues vernaculaires a introduit dès la fin du vII ${ }^{\mathrm{e}}$ ou le début du vIII ${ }^{\mathrm{e}}$ siècle une séparation des mots ${ }^{14}$. Dans

11. On ne doit pas oublier, bien évidemment, les autres supports : peinture (comme dans la crypte de Saint-Germain à Auxerre, CIFM, t. 21, no 45-53, p. 42-53), mosaïque (comme à Germigny-des-Prés dans le Loiret) ou ivoire, par exemple ; mais ceux-ci sont minoritaires dans l'ensemble du corpus conservé.

12. Sidoine Apollinaire, Lettres, éd. et trad. André Loyen, Paris, 1970, t. II, p. 101-103, lettre à Secundus (469), $\S 4:$ : Posco ut [...] tua cura sed meo sumptu resurgat in molem sparsa congeries, quam levigata pagina tegat. "

13. Parmi de multiples exemples, on peut citer le commentaire carolingien de Priscien dans le ms. 2 de Berne, fol. 136 : «Littera est proprie nota elementi et velut imago vocis. " Grammatici Latini, éd. Hermann Hagen, t. VIII, Leipzig, 1880, p. CLXXXI.

14. Paul Saenger, Space between words : the origins of silent reading, Stanford, 1997 (Figurae), réimpr. 2000, et bref exposé du même, "Coupure et séparation des mots sur le continent au Moyen Âge ", dans Mise en page et mise en texte du livre manuscrit, dir. Henri-Jean Martin et Jean 
l'empire carolingien, les instructions d'Alcuin aux copistes de Saint-Martin de Tours établissent en outre une relation très nette entre la lecture à haute voix et la nécessité d'une ponctuation raisonnée ${ }^{15}$; celle-ci se développera de manière plus rapide et plus intense dans la pratique manuscrite que dans les inscriptions, qui n'en adoptent l'usage qu'à partir du XI ${ }^{\mathrm{e}}$ siècle. Est-ce à dire que la lecture des inscriptions, jusque vers l'an mille, reste l'apanage des clercs, nécessaires intermédiaires dans la transmission du contenu du texte? La question, à défaut d'être résolue, vaut au moins la peine d'être posée.

Si le caractère compact et continu des inscriptions est récurrent jusqu'au $\mathrm{XI}^{\mathrm{e}}$ siècle, on observe en revanche des variations graphiques importantes dans le haut Moyen Âge. L'époque mérovingienne oscille entre le legs antique, luimême rendu pluriel par les traditions impériales ou damasiennes, et des nouveautés graphiques propres à chaque aire culturelle. Dans des productions épigraphiques de très haut niveau, comme certaines inscriptions de dédicace d'autel, à Minerve ou Le Ham par exemple ${ }^{16}$, on utilise volontiers la capitale monumentale romaine, officielle et prestigieuse. Malheureusement, la disparition quasi totale de tous les monuments de cette période nous prive de ce type de documents et rend d'autant plus visible l'autre tradition graphique liée à une pratique plus personnelle, ou plus quotidienne. Issues pour la plupart des capitales romaines, les lettres des inscriptions mérovingiennes abandonnent toutefois leur belle rotondité et adoptent un module extrêmement allongé. Elles cultivent plus volontiers l'angle droit que la courbe et il n'est pas rare de voir un $C$ carré ou un $O$ en losange. Elles sont disposées l'une après l'autre, suivant tout au plus une réglure simple et parfois très fugitive mais sans se conformer à une stricte ordinatio. Les hastes verticales dépassent régulièrement du point de jonction des courts traits horizontaux dans le $C$ carré, le $E$, le $F$, voire le $L$, et des petites panses des $B, P$ et $R$; les traits horizontaux n'hésitent en outre pas à obliquer vers le bas, notamment dans le $L$, ou vers le haut, dans le $F$ comme on le voit bien, par exemple, sur les inscriptions mérovingiennes découvertes récemment dans les fouilles du baptistère de Brioude ${ }^{17}$ (ill. 1). L’irrégularité enfin est souvent de mise, de manière certes

Vezin, Paris, 1990, p. 451-455 (voir p. 451). Voir encore, sur ce sujet, Jeannette Moreau-Maréchal, "Recherches sur la ponctuation ", dans Scriptorium, 22, 1968, p. 56-66 ; J. Vezin, "La ponctuation du VIII ${ }^{\mathrm{e}}$ au XII ${ }^{\mathrm{e}}$ siècle ", dans Mise en page et mise en texte..., p. 439-442 ; et Malcolm B. Parkes, Pause and effect : an introduction to the history of punctuation in the West, Aldershot, 1992. Mots et groupes de mots sont déjà bien séparés par des points dans les inscriptions de la célèbre croix de Ruthwell, du milieu du vin' ${ }^{\mathrm{e}}$ siècle (France et Peter Sharrott, Écosse romane, La Pierrequi-Vire, 1985, p. 32-35, pl. 1-2).

15. Le titulus rédigé par Alcuin pour le scriptorium tourangeau est très clair : «Per cola distinguat proprios et commata sensus | et punctos ponant ordine quosque suo | ne vel falsa legat, taceat vel forte repente | ante pios fratres lector in ecclesia. " MGH., Poet. Lat., t. I, $\mathrm{n}^{\circ}$ XCIV, p. 320.

16. E. Le Blant, Les inscriptions chrétiennes..., resp. t. II, $\mathrm{n}^{\circ} 609$, p. 428-451, et t. I, n ${ }^{\circ} 91$, p. 185-186.

17. Éd. Fabrice Gauthier, «Inscriptions paléochrétiennes découvertes dans le baptistère SaintJulien de Brioude (Haute-Loire) ", dans Hortus artium medievalium, t. 10, 2004, p. 211-215. 


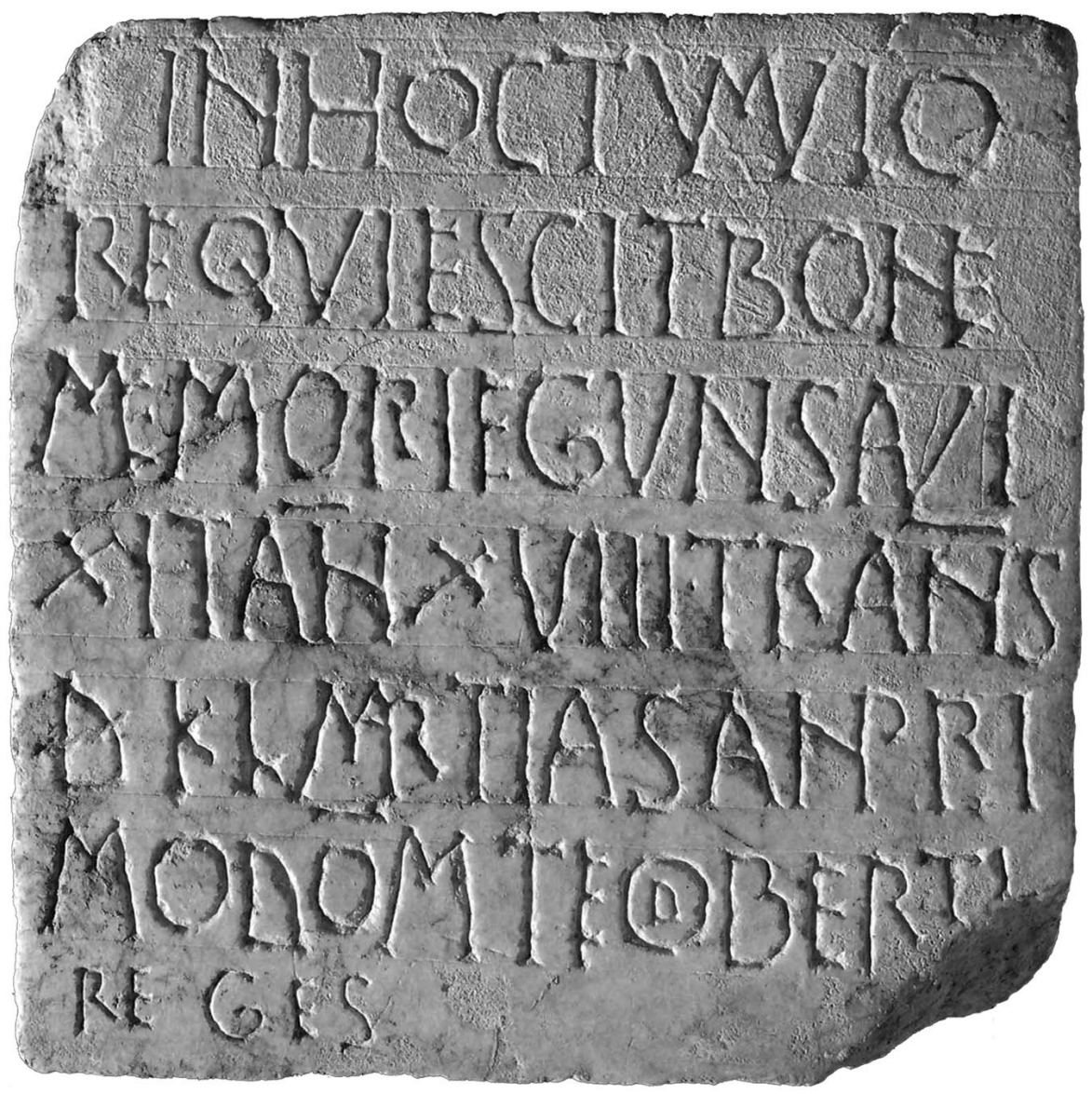

ILL. 1. - Inscription funéraire mérovingienne. Brioude (Haute-Loire), baptistère. Cliché F. Gauthier.

variable mais récurrente, dans la graphie et le module des lettres comme dans leur mise en page.

Cette tradition graphique mérovingienne, à l'instar des manifestations linguistiques contemporaines, a été diversement appréciée des chercheurs. Mesurée à l'aune du classicisme impérial romain (réel ou supposé), elle a été la plupart du temps méprisée comme signe d'une dégradation de l'art d'écrire, liée à la décadence supposée de la civilisation de l'Occident romain sous l'effet néfaste des barbares. Cependant, l'évolution de l'écriture des inscriptions mérovingiennes peut tout aussi bien être considérée comme témoignage de la vitalité d'une culture écrite indépendante de l'ambiance compassée des grands scriptoria. La tradition épigraphique de l'Antiquité tardive semble s'être enrichie au cours de l'époque mérovingienne par le développement spontané de 
nouvelles formes de lettres, peut-être inspirées par le tracé des runes, langage graphique dont on trouve des traces plus ou moins fugitives sur le continent ${ }^{18}$.

Même si les témoignages ne sont pas très nombreux, l'apogée de ce mouvement de création graphique semble se situer au vIII siècle, plus particulièrement dans la seconde moitié ; que la technique soit lapidaire, comme dans les épitaphes angevines sur ardoise ${ }^{19}$, ou picturale, comme sur les stucs de Vouneuil-sous-Biard ${ }^{20}$, les tracés deviennent plus vigoureux, plus anguleux ; les courbes des $C, G$ ou $O$ se brisent, aboutissent parfois même à des formules graphiques uniques et sans postérité, comme les $G$ de l'inscription d'Autbertus (ill. 2) à Angers ${ }^{21}$. Le caractère heurté de l'écriture est renforcé par une accentuation des traits de réglure qui prennent désormais une part importante dans la structuration visuelle du texte et par l'adjonction systématique de triangles décoratifs à l'extrémité des traits de structure, exagérant à l'extrême l'usage habituel des apices. Quelques décennies avant la réforme de l'écriture promue par Charlemagne, nul doute que les lapicides ne répondent déjà à la demande de savants qui aiment les lettres et jouent avec elles. L'inscription de Saint-Gaudens ${ }^{22}$, avec ses enclavements multiples et la complexité de sa composition générale reposant sur une lecture en zigzag, le prouve aisément. La tradition des jeux graphiques par conjonction, enclavement ou entrelacement de lettres perdure au $\mathrm{Ix}^{\mathrm{e}}$ siècle, comme le montre par exemple l'épitaphe d'Amelius, mort en 874 à Poitiers ${ }^{23}$. Elle perd toutefois de son importance, voire devient marginale face à une écriture qui puise ses canons dans l'épigraphie impériale romaine.

\section{L'ÉPIGRAPHIE CAROLINGIENNE.}

L'extrême fin du vIII ${ }^{\mathrm{e}}$ et le début du IX ${ }^{\mathrm{e}}$ siècle marquent en effet une rupture importante dans les pratiques scripturales, qu'elles soient manuscrites ou épigraphiques. Dans le contexte d'unification et de romanisation du royaume carolingien qui deviendra empire en 800 , les scriptoria adoptent, selon un

18. On peut signaler à ce titre le projet européen d'inventaire mené par Svante Fischer, présenté dans " A survey of runic inscriptions : the IRF-RIF Project, 2006-2007 (Inscriptions runiques de France)", dans Bulletin de liaison de l'AFAM, $\mathrm{n}^{\circ} 30,2006: 27^{\text {es }}$ Journées internationales d'archéologie mérovingienne, Caen, 29 sept.-1 ${ }^{\text {er }}$ oct. 2006, p. 45-46.

19. Épitaphes d'Autbertus, en 784 (dessinée dans Daniel Prigent et Jean-Yves Hunot, La mort, voyage au pays des vivants : pratiques funéraires en Anjou [expos.], Angers, 1996, p. 35), de Balthadus et d'Erdramnus, en 771, découvertes à Saint-Martin d'Angers et encore inédites.

20. En attendant l'article de Cécile Treffort, «Les stucs découverts à Vouneuil-sous-Biard : le dossier épigraphique ", dans Les stucs de l'Antiquité du site de Vouneuil-sous-Biard, dir. Christian Sapin, Bordeaux, numéro spécial de Gallia, on peut se reporter à la notice de la même, "Les inscriptions de Vouneuil », dans Le stuc : visage oublié de l'art médiéval (expos., Poitiers, musée Sainte-Croix, 2004-2005), introd. C. Sapin, Paris-Poitiers, 2004, p. 85-86.

21. Cf. note 19 .

22. CIFM, t. 7, 1982, no 40, p. 76-77 et fig. 43.

23. CIFM, t. 1, 1974, no 83, p. 100-102 et fig. 37. 


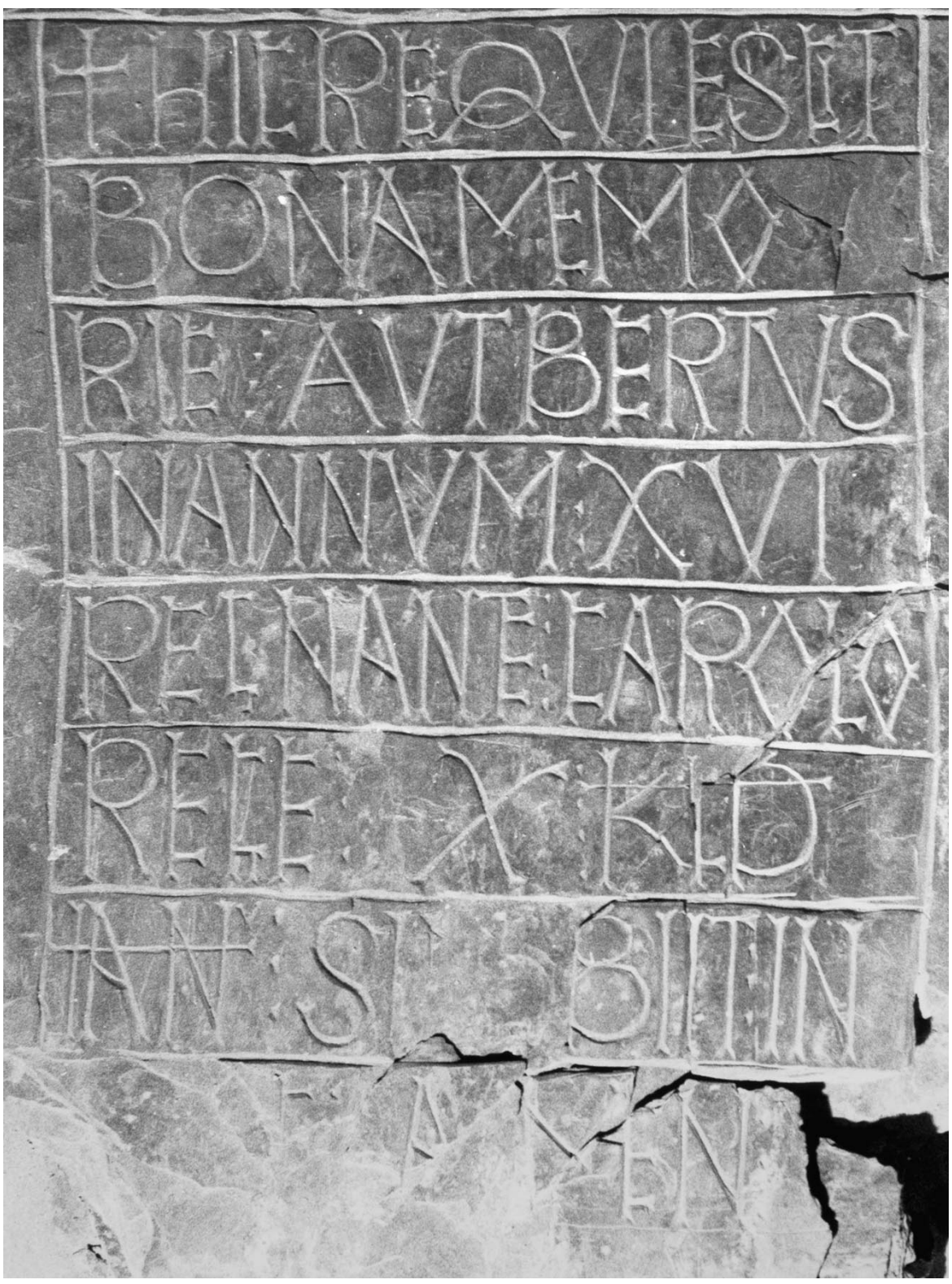

ILL. 2. - Inscription funéraire d'Autbertus, 784. Angers (Maine-et-Loire), service départemental de l'Archéologie, n ${ }^{\circ}$ SM-L.01l (prov. Saint-Martin d'Angers). Cliché J. Michaud (сеscm/CIFM). 
rythme et des modalités variables, une nouvelle écriture : la minuscule caroline. S'affranchissant des écritures locales antérieures, cette nouvelle écriture apparaît clairement comme un instrument d'unification politico-religieuse. Les écrits d'Alcuin, qui confirment la part de l'initiative royale, mettent en lumière les enjeux multiples du soin particulier que les scribes doivent apporter à la réalisation des manuscrits, à la copie des textes, à leur ponctuation ${ }^{24}$. Aucun document ne vient en revanche éclairer la réforme épigraphique, bien réelle pourtant, si ce n'est les inscriptions elles-mêmes. Dès la fin du vIII siècle, quelques inscriptions particulièrement prestigieuses adoptent une écriture nettement "romanisante », par référence à la Rome impériale ${ }^{25}$ : usage de la capitale monumentale classique - appelée uncialis dans les textes carolingiens ${ }^{26}$, absence totale de séparation entre les mots et de ponctuation, ordinatio extrêmement géométrique, abréviations réservées à la datation et, surtout, aux nomina sacra ${ }^{27}$. À côté des épitaphes épiscopales de Megingoz à Wurtzbourg ou de Witgar à Augsbourg ${ }^{28}$, qui témoignent du courant de redécouverte de l'Antiquité, l'exemple le plus achevé de cette réforme est sans conteste l'épitaphe d'Hadrien $\mathrm{I}^{\mathrm{er}}$ réalisée sur ordre de Charlemagne après la mort du pontife en $795^{29}$. Véritable hapax par sa perfection technique et calligraphique,

24. Sur la période, voir en dernier lieu Marie-Pierre Laffitte et Charlotte Denoël, collab. Marianne Besseyre, Trésors carolingiens : le livre manuscrit de Charlemagne à Charles le Chauve (expos., Bibl. nat. de Fr., 2007), Paris, 2007 ; Cécile Treffort, Mémoires carolingiennes : l'épitaphe entre célébration mémorielle, genre littéraire et manifeste politique (milieu du VIII -début du $X I^{e}$ s.), Rennes, 2007 (Histoire), notamment p. 142-156, "Écriture et calligraphie ».

25. Pour les caractéristiques de l'écriture épigraphique (italienne) à l'époque carolingienne : Nicolete Gray, «The palaeography of Latin inscriptions in the eighth, ninth and tenth centuries in Italy ", dans Papers of the British School at Rome, t. 16, 1948, p. 38-171 ; Felice Grossi Gondi, "Excursus sulla paleografia medievale epigrafica del sec. IX ", dans Dissertazioni della Pontificia Accademia romana di archeologia, $2^{\text {e }}$ sér., t. 13, 1918, p. 147-179 et pl.

26. Voir notamment Bernhard Bischoff, «Die alten Namen der lateinischen Schriftarten », dans id., Mittelalterliche Studien : ausgewählte Aufsätze zur Schriftkunde und Literaturgeschichte, Stuttgart, 1966, t. I, p. 1-6, ainsi que les réflexions de David Ganz, "Roman books reconsidered : the theology of Carolingian display script ", dans Early medieval Rome and the Christian West : essays in honour of Donald A. Bullough, ed. Julia M. H. Smith, Leyde-Boston-Cologne, 2000, p. 297-315.

27. Sur le sujet, voir l'ouvrage ancien mais non remplacé de Ludwig Traube, Nomina sacra : Versuch einer Geschichte der christlichen Kürzung, Munich, 1907.

28. Pour l'épitaphe de Megingoz, voir en particulier Franz Xaver Herrmann, «Die Versinschrift für Bischof Megingoz († 794) im Neumünster zu Würzburg», dans Würzburger Diözesangeschichtsblätter, t. 48, 1986, p. 133-162; l'épitaphe de Witgar a quant à elle été éditée par B. Bischoff, « Die karolingischen Inschriftensteine aus der Krypta-Grabung 1961/1962 », dans Die Ausgrabungen in St. Ulrich und Afra in Augsburg, 1961-1968, éd. Joachim Werner, Munich, 1977 (Münchner Beiträge zur Vor- und Frühgeschichte, t. 23), p. 264-266 et pl. 82-83, puis Wolfgang Dieter Lebek, "Das Versepitaph des Augsburger Bischofs und königlichen Kanzlers Witgar (gest. 887) ", dans Zeitschrift des historischen Vereins für Schwaben, t. 75, 1981, p. 73-85.

29. Parmi les nombreuses études consacrées à cette inscription, retenons ici les trois principales : Giovanni Battista de Rossi, "L'inscription du tombeau d'Hadrien I ${ }^{\mathrm{er}}$ composée et gravée en France par ordre de Charlemagne ", dans École française de Rome, Mélanges d'archéologie et d'histoire, t. 8, 1888, p. 478-501 ; Luitpold Wallach, "Alcuin's epitaph of Hadrian I : a study in Carolingian epigraphy ", dans American journal of philology, t. 72, 1951, p. 128-144, rééd. dans 


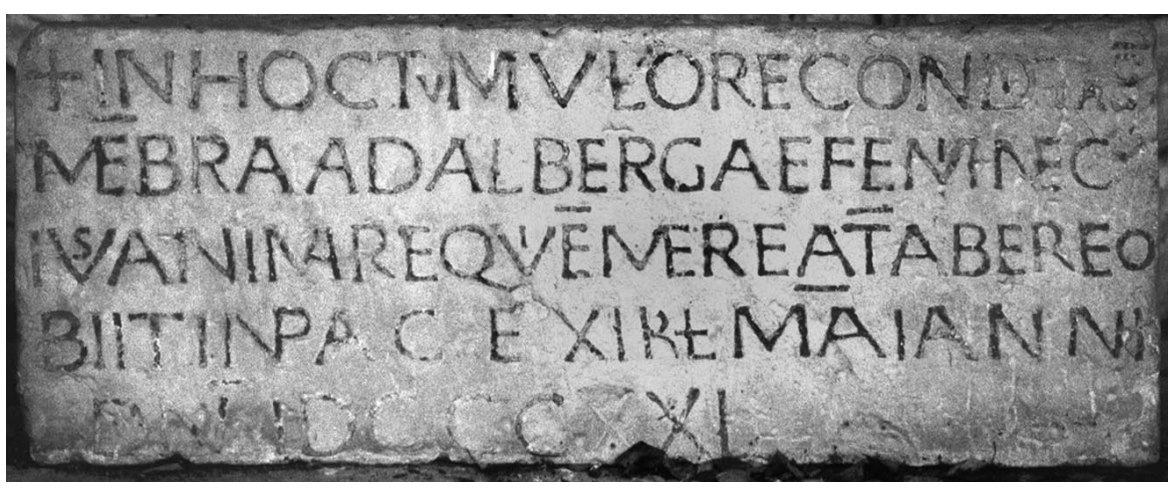

ILL. 3. - Inscription funéraire d'Adalberga, 830 ou 840. Tours (Indre-et-Loire), Société archéologique de Touraine, $\mathrm{n}^{\circ}$ inv. HG 950.5.1. Cliché C. Treffort.

elle semble tracer la voie de la renaissance épigraphique qui se diffuse dans les décennies suivantes à travers tout l'empire carolingien.

En France, les preuves irréfutables de l'adoption du modèle d'écriture romanisante sont perceptibles à partir du règne de Louis le Pieux, notamment à Tours et Angers où, par bonheur pour l'épigraphiste, les inscriptions sont datées ; on peut citer par exemple l'épitaphe d'Adalberga à Tours (ill. 3), de 830 ou $840^{30}$, dont l'écriture adopte un module très large pour des capitales monumentales romaines ; l'incrustation de plomb interdit au lapicide de donner aux lettres l'élégance qui caractérise à l'inverse l'épitaphe de l'abbé Ato (ill. 4), mort à Angers en $835^{31}$. Cette dernière, sculptée avec finesse sur une grande dalle d'ardoise, est d'autant plus caractéristique de la réforme carolingienne que son texte est composé d'un centon empruntant ses vers exclusivement au poème d'Alcuin sur l'église d'York ${ }^{32}$. L'ordinatio de l'ensemble est parfaite : dans un cadre général, on trouve un texte principal, métrique, disposé en huit lignes correspondant aux huit vers, et un cartouche latéral, encadré de manière indépendante, destiné à la date. La même réglure double sert pour les

id., Alcuin and Charlemagne, New York, 1959, chap. x, p. 178-197 ; et Sebastian Scholz, « Karl der Große und das "Epitaphium Hadriani ", ein Beitrag zum Gebetsgedenken der Karolinger », dans Das Frankfurter Konzil von 794, Kristallisationspunkt karolingischer Kultur : Akten zweier Symposien (vom 23. bis 27. Februar und vom 13. bis 15. Oktober 1994), éd. Rainer Berndt, t. I : Politik und Kirche, Mayence, 1997 (Quellen und Abhandlungen zur Mittelrheinischen Kirchengeschichte, 80/1), p. 373-394.

30. Inscription appartenant à la Société archéologique de Touraine, $\mathrm{n}^{\circ}$ inv. HG 950.5.1; citée par G. B. de Rossi, «L'inscription du tombeau d'Hadrien $\mathrm{I}^{\mathrm{er}} \ldots$ »., p. 489.

31. Sur cette inscription conservée à Angers, musée des Antiquités, nº inv. GF 24, on pourra consulter C. Treffort, "Un témoin de la vie politique et culturelle carolingienne à Angers : l'épitaphe sur ardoise de l'abbé Ato († 835) », dans les actes du colloque "L'Anjou au haut Moyen Âge ", éd. Noël-Yves Tonnerre, Angers, à paraître.

32. Alcuin, Poème sur l'Église d'York, dans MGH, Poet. Lat., t. I, p. 169-206. 
ILL. 4. - Inscription funéraire d'Ato, 835. Angers (Maine-et-Loire), musée des Antiquités (réserves Notre-Dame), no GF 24. Cliché J. Michaud (СЕscm/CIFM).

deux parties, le texte latéral étant gravé en plus petites lettres entre les lignes qui structurent l'interlignage du texte principal. La hiérarchie graphique révèle celle des textes tandis que l'ordinatio commune illustre leur complémentarité. Là encore, le module des lettres, relativement large, est régulier. La composition graphique est dense, sans séparation de mots ni ponctuation, et utilise exclusivement des capitales monumentales classicisantes (mis à part le $D$ oncial de la date).

En France, la tradition épigraphique romanisante semble atteindre la perfection dans le deuxième quart du $\mathrm{Ix}^{\mathrm{e}}$ siècle, ce qui suggère à la fois une diffusion plus large du modèle graphique et une volonté plus affirmée de l'adopter. Les "lettres antiques" appelées "onciales" dont Loup de Ferrières sollicite la mesure et la forme du scribe royal Bertcaudus par l'intermédiaire d'Einhard de Seligenstadt en $836^{33}$ sont celles qui servent conjointement aux tituli des manuscrits et aux inscriptions peintes ou sculptées de l'époque carolingienne. L'alphabet modèle conservé dans le manuscrit 250 de Berne ${ }^{34}$, considéré par Bernhard Bischoff comme celui qui fut envoyé par Einhard à Loup en réponse à sa lettre, est significatif de l'attention portée, en ce deuxième quart du $\mathrm{IX}^{\mathrm{e}}$ siècle, au tracé des lettres épigraphiques et de leur enjeu politique : l'écriture romanisante est, sans conteste possible, une écriture officielle et impériale dont l'usage est loin d'être anodin. Il faut d'ailleurs signaler, pour renforcer cette impression, que les inscriptions contemporaines d'un moindre niveau d'exécution ne satisfont pas obligatoirement aux critères de régularité, de monumentalité et de romanité évoqués précédemment.

33. «Praeterea scriptor regius Bertcaudus dicitur antiquarum litterarum, dumtaxat earum quae maximae sunt et unciales a quibusdam vocari existimantur, habere mensuram descriptam, itaque si penes vos est, mittite mihi eam per hunc, queso, pictorem, cum redierit, scedula tamen diligentissime sigillo munita." Loup de Ferrières, Correspondance, éd. et trad. Léon Levillain, Paris, 1927 (Les classiques de l'histoire de France au Moyen Âge), n 5.

34. Bern, Burgerbibliothek, ms. 250, fol. 1lv ; folio reproduit dans 799 : Kunst und Kultur der Karolingerzeit, Karl der Grosse und Papst Leo III. in Paderborn (expos., Paderborn 1999), éd. Christoph Stiegemann et Matthias Wemhoff, Mayence, 1999, p. 335 (notice VI.13) et 336 (ill.), avec commentaire. 
Si les capitales romaines, à partir de la réforme carolingienne, restent en usage dans les inscriptions pendant plusieurs siècles, elles voient leur module s'allonger progressivement à partir du milieu du $\mathrm{IX}^{\mathrm{e}}$ et sont concurrencées par d'autres formes. Dès la seconde moitié $d u \mathrm{IX}^{\mathrm{e}}$ siècle, la présence de lettres carrées, d'abord timide, s'intensifie puis elle se systématise au $\mathrm{x}^{\mathrm{e}}$ siècle. On observe une évolution similaire pour les lettres onciales, notamment le $D$ et le $E$, dont la fréquence augmente au cours des décennies. Le rythme et l'ampleur de cette évolution varient selon les lieux, rejetant dans le passé la belle unité rêvée par Charlemagne. Elle est en revanche parallèle à celle qui marque les tituli manuscrits. Une étude conjointe des inscriptions et des codices datés et localisés reste à entreprendre : elle serait du plus grand intérêt car elle permettrait de caractériser mieux que nous ne pouvons le faire actuellement l'écriture épigraphique $\mathrm{du} \mathrm{x}^{\mathrm{e}}$ siècle. Rares sont pour cette période les inscriptions datées ou datables comme l'épitaphe de Salomon à Poitiers (ill. 5), vers 976-980, avec sa belle écriture carrée ${ }^{35}$. Les témoignages épigraphiques attribués au $\mathrm{x}^{\mathrm{e}}$ siècle en France le sont en général par des arguments historiques ou par comparaison stylistique, critères certes indispensables, mais qui posent, une fois de plus, le problème des méthodes de comparaison et des inscriptions de référence. Bien du travail reste encore à faire pour remédier à l'évanescence épigraphique au $\mathrm{x}^{\mathrm{e}}$ siècle, qui contraste nettement avec les siècles suivants.

\section{Le RENOUVEAU DES XI ${ }^{\mathrm{e}}$ ET XII ${ }^{\mathrm{e}}$ SIÈCLES : ÉLÉGANCE ET LISIBILITÉ.}

La tradition graphique des inscriptions connaît en effet une inflexion très nette aux XI ${ }^{\mathrm{e}}$ et XII ${ }^{\mathrm{e}}$ siècles ; celle-ci se caractérise d'abord par un souci de clarté, qui va rompre avec la présentation compacte d'une "écriture continue» des temps précédents, puis, à partir du XII ${ }^{\mathrm{e}}$ siècle principalement, par une nouvelle recherche d'élégance. Si ces deux derniers traits peuvent être présentés avec assurance, on devra toujours tenir compte de ce que cette évolution ne suit pas une progression parfaitement linéaire et garder à l'esprit qu'il faut considérer et la région, et le support, et le lieu de production de l'inscription pour apporter de nécessaires nuances au tableau d'ensemble.

L'évolution tient à l'usage du latin, devenu langue savante, qui entraîne la nécessité d'introduire dans les textes espacements, ponctuation et autres éléments pour faciliter la lecture ${ }^{36}$ quand, avec le développement des écoles capitulaires et la naissance des universités, elle ne sera plus le privilège d'une élite restreinte ; quand la versification sera considérée comme le seul style noble et que la poésie rythmique se combinera avec la poésie métrique ${ }^{37}$ (vers léonin simple ou riche), d'où des textes plus longs, plus élaborés, moins prévisibles

35. CIFM, t. 1, p. 59-61 et fig. 38 .

36. Voir note 14 .

37. Jacques Paul, Histoire intellectuelle de l'Occident médiéval, Paris, 1973, p. 231. 


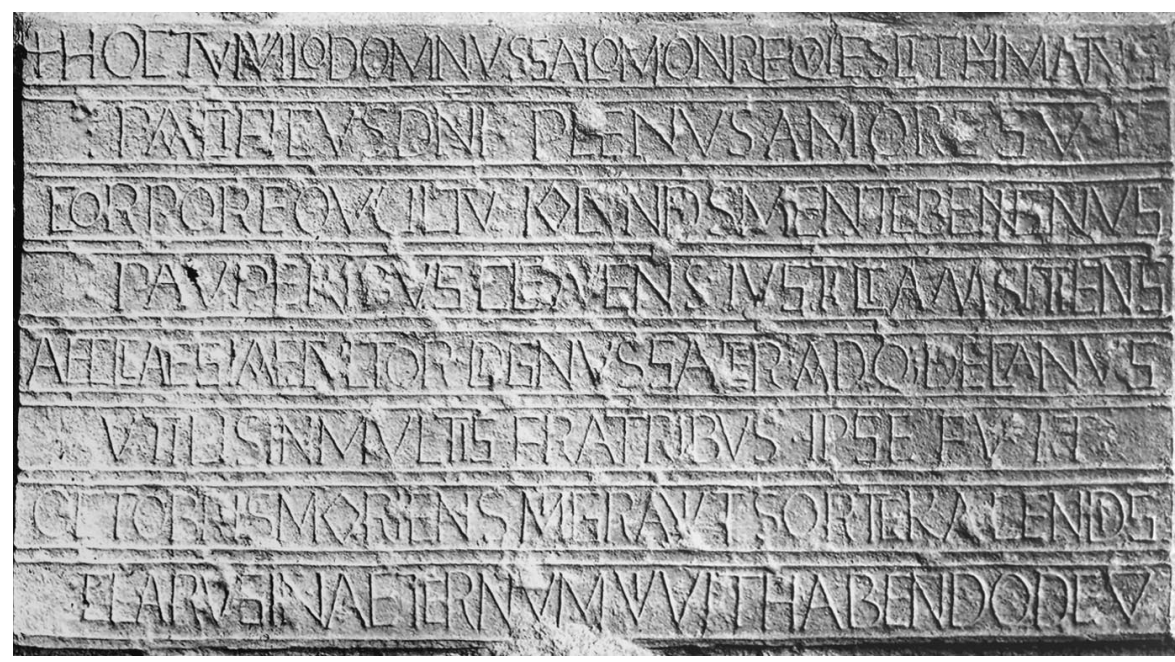

ILL. 5. - Inscription funéraire de Salomon, 976-980. Poitiers (Vienne), Saint-Hilaire, déambulatoire, mur sud. Cliché J. Michaud (CESCM/CIFM).

pour le lecteur; quand les écrits se diversifieront en traités scientifiques, ouvrages de médecine, d'astronomie, de sciences naturelles et autres collections canoniques ${ }^{38}$.

Le souci de faciliter la lecture se marque d'abord par la ponctuation ${ }^{39}$ et les espacements, qui s'installent progressivement dans les inscriptions au cours du $\mathrm{XI}^{\mathrm{e}}$ siècle et seront presque de règle au XII ${ }^{\mathrm{e}}$. Au linteau de l'église de Saint-Genisdes-Fontaines (Pyrénées-Orientales), une inscription indique que «l'abbé Guillaume a fait faire cette œuvre" en 1019-1020 40 : l'écriture est assez irrégulière bien que la pierre ait fait l'objet d'une réglure ; il n'y a ni espacement des mots, ni ponctuation, et les liaisons de lettres et abréviations ajoutent au caractère compact du texte. Pas de ponctuation non plus dans l'inscription d'Éguilles (Bouches-du-Rhône) en $1026^{41}$ mais des espacements marquent l'indication du jour de la dédicace. L'épitaphe d'Étienne à Saint-André-le-Bas de Vienne (Isère) en $1012^{42}$ est quant à elle soignée : cadre, double réglure pour

38. La ponctuation apparaît indispensable dans des textes comme la table pascale du xiI ${ }^{\mathrm{e}}$ siècle à Saint-Étienne-de-la-Cité de Périgueux (éd. CIFM, t. 5, p. 28-31 et fig. 13) ou l'énumération des reliques sur le reliquaire de Bouillac provenant de l'abbaye de Grandselve (éd. CIFM, t. 8, p. 117-125 et fig. 93, 94, 97).

39. Le frère Philippe-Martin Hubert a donné un recueil des textes relatifs à la ponctuation, d'Aristote et Cicéron jusqu'au XII ${ }^{\mathrm{e}}$ siècle, "Corpus stigmatologicum minus ", dans Archivum latinitatis Medii Aevi, t. 37, 1970, p. 14-169, et "Traité du vocabulaire de la " ponctuation " aux temps médiévaux : un cas d'incertitude lexicale ", ibid., t. 38, 1972, p. 57-166.

40. CIFM, t. 11, p. 138-139 et fig. 106.

41. CIFM, t. 14, p. 89-91.

42. CIFM, t. 15, 1990, n 55 , p. 58-59 et fig. 36 . 


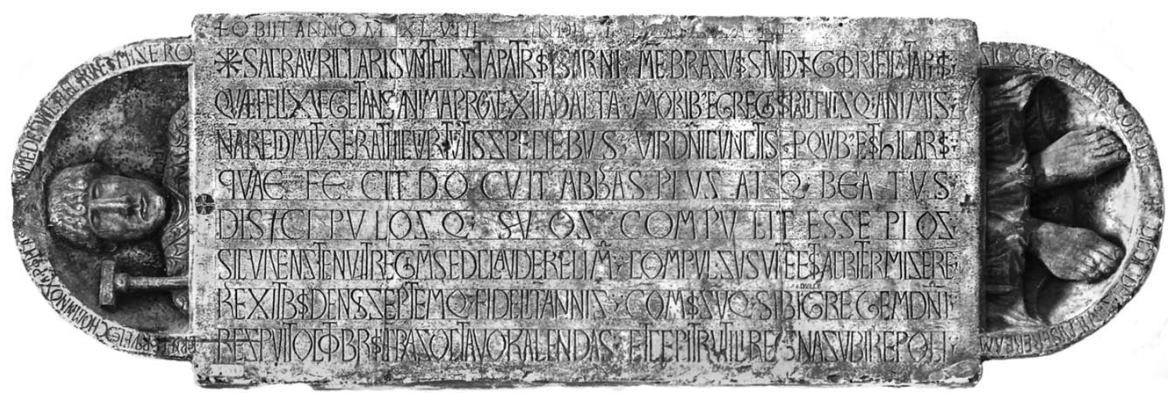

ILL. 6. - Inscription funéraire d'Isarn, 1048. Marseille (Bouches-du-Rhône), Saint-Victor. Cliché J. Michaud (CESCM/CIFM).

chaque ligne ${ }^{43}$ et une ponctuation par deux points verticaux signalant chacun des cinq hexamètres par ailleurs gravés ligne à ligne; il n'y a toutefois pas d'espacement entre les mots et le grand nombre de lettres enclavées n'est pas fait pour faciliter la lecture. Dans l'épitaphe de l'abbé Hugues, toujours à Saint-André-le-Bas, en $1032^{44}$, les vers sont séparés par trois points en triangle ; là aussi, il y a beaucoup de ligatures, enclavements et entrelacements de lettres. Dans la remarquable épitaphe de l'abbé Isarn à Saint-Victor de Marseille en $1048(i l l .6)^{45}$, les trois premières et les trois dernières lignes correspondent à deux vers, que sépare une ponctuation par deux points au-dessus d'une virgule, et il n'y a pas d'espacement entre les mots ; en revanche, on a gravé un seul vers dans chacune des deux lignes centrales et là, les mots sont parfaitement séparés. L'inscription de 1069 sur la plaque de gauche de l'autel majeur en la cathédrale d'Elne (Pyrénées-Orientales) ${ }^{46}$ a une ponctuation par un, deux ou trois points séparant des groupes de mots, ou parfois des mots, sans une évidente cohérence. À la fin de trois lignes, les mots sont coupés; il n'y a pas d'espacement entre les mots à l'intérieur de chaque groupe de mots et les liaisons de lettres très nombreuses gênent une appréhension rapide du texte (ill. 7). Dans la belle inscription de dédicace de Moissac (Tarn-et-Garonne) en $1063{ }^{47}$, les mots sont séparés par des points, les vers par deux points verticaux, mais dans l'inscription de dédicace de la proche abbatiale de Saint-Maurin (Lot-et-Garonne) en $1097^{48}$, il n'y a pas de ponctuation et les espacements sont rares.

43. La pratique de la réglure dans les inscriptions mériterait examen. Pour les manuscrits, voir à titre d'initiation le chapitre que lui consacre Jacques Lemaire dans Introduction à la codicologie, Louvain-la-Neuve, 1989 (Publications de l'institut d'études médiévales, Textes, études, congrès, 9), p. 109-125.

44. CIFM, t. 15, p. 58-61 et fig. 36-37.

45. CIFM, t. 14, p. 99-102 et fig. 63.

46. CIFM, t. 11, p. 54-57 et fig. 37 .

47. CIFM, t. 8, p. 131-134 et fig. 111.

48. CIFM, t. 6, p. 195. 


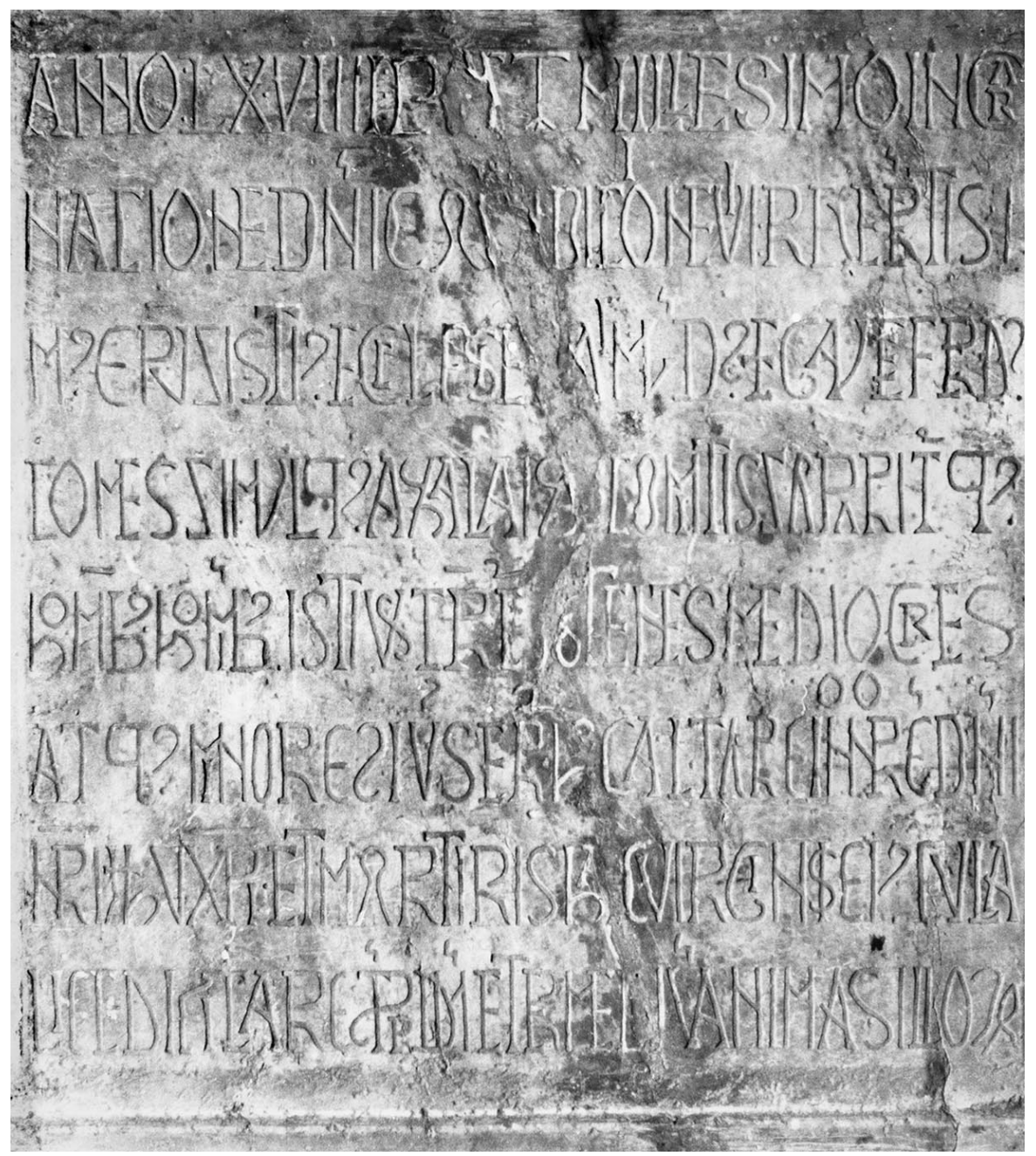

ILL. 7. — Notice de construction d'un autel, 1069. Elne (Pyrénées-Orientales), cathédrale, plaque de gauche. Cliché L. Michaud (CEscm/CIFM).

$\mathrm{Au} \mathrm{XII}^{\mathrm{e}}$ siècle, on trouve encore quelques exemples d'inscriptions sans espacements ni ponctuation et des ponctuations irrégulières ou par groupes de mots; la régularité progresse toutefois et au dernier tiers du XII ${ }^{e}$ siècle la ponctuation est presque la règle, parfois remplacée par de simples espacements. En épigraphie, ce sont principalement les points, simples ou verticaux, qui marquent cette ponctuation. On peut aussi trouver, comme dans les manuscrits, une ponctuation par point-et-virgule, point-et-virgule inversé, deux points surmontant une virgule, comme dans l'épitaphe de Bernard, 
sacriste de Saint-Paul de Narbonne (1151), au musée des Augustins de Toulouse ${ }^{49}$.

Abbon de Fleury, à la fin du $\mathrm{x}^{\mathrm{e}}$ siècle, avertit d' "éviter la confusion qui se produit d'habitude entre deux mots, soit dans la prononciation soit dans l'écriture " ${ }^{50}$ : de fait, l'abbaye de Fleury fut, à son époque, « un des centres à partir desquels la séparation, inspirée des modèles anglo-saxons, commença son rayonnement sur la France, l'Allemagne, l'Italie " ${ }^{51}$. Les inscriptions tumulaires des $\mathrm{XI}^{\mathrm{e}}$ et $\mathrm{XII}^{\mathrm{e}}$ siècles à Saint-Benoît-sur-Loire ${ }^{52}$ ont effectivement toutes un signe de ponctuation entre chaque mot ou groupe de mots (in Christo, in pace pour l'abbé Véran, vel quid pour l'abbé Joscerand, in mundo ou sobrius et castus dans la quatrième inscription). On aimerait pouvoir faire des comparaisons avec la France du nord mais il faudra attendre pour cela une couverture photographique. En Normandie, on ne dispose pas d'inscriptions bien datées pour le $\mathrm{XI}^{\mathrm{e}}$ siècle ; on trouve habituellement une ponctuation au XII ${ }^{\mathrm{e}}$ siècle, si ce n'est que l'épitaphe sur plomb d'un abbé Reinfredus en $1150{ }^{53}$ vient nous rappeler qu'il peut toujours y avoir des exceptions, car l'écriture y est irrégulière, les coupures de mot en fin de ligne maladroites (par exemple : huju/s) et il n'y a ni ponctuation ni espacement. Les coupures de mot se rencontrent à toutes les époques et même les inscriptions les plus soignées ne les évitent pas toujours. Le trait d'union apparaît en Angleterre à la fin $\mathrm{du} \mathrm{x}^{\mathrm{e}}$ siècle. Son emploi, qui ne se généralisera qu'un siècle plus tard, témoigne de la conscience prise par les copistes et les lecteurs de l'individualité des mots ${ }^{54}$; mais il est très rare en épigraphie. La broderie de Bayeux en offre du moins un bel exemple, lorsque Harold s'embarque pour la côte normande 55 : ET vELIs : VENTO : PLENIS VE =/= NIT : IN : TERRA : Le même souci de clarté introduit au $\mathrm{XI}^{\mathrm{e}}$ siècle des accents sur deux $i$ qui se suivent : c'est le cas à Toulouse en $1151^{56}$, dans le mot petiit, mais, là encore, les exemples sont rares en épigraphie.

La lisibilité du texte, c'est aussi la réglure, et le texte est évidemment bien mieux ordonné lorsque celle-ci est double, permettant de placer les signes d'abréviation dans l'intervalle ainsi créé entre les lignes. On trouve la double réglure dès le $\mathrm{x}^{\mathrm{e}}$ siècle ; mais dans les inscriptions datées, c'est dans la seconde moitié du XII siècle que se multiplient les exemples, ainsi à Toulouse ou à Vienne. Il faudrait y réserver une étude particulière.

49. CIFM, t. $7, \mathrm{n}^{\circ} 48$, p. $86-88$ et fig. $52-53$.

50. Abbo Floriacensis, Quaestiones grammaticales, éd. Anita Guerreau-Jalabert, Paris, 1982 (Auteurs latins du Moyen Âge), p. 245.

51. P. Saenger, "Coupure et séparation des mots... », p. 452.

52. Boucher de Molandon, "Inscriptions tumulaires des $\mathrm{XI}^{\mathrm{e}}$ et $\mathrm{XII}^{\mathrm{e}}$ siècles à Saint-Benoît-surLoire ", dans Mémoires de la Société archéologique et historique de l'Orléanais, t. 18, 1881, p. 527.

53. CIFM, t. 22, n 245 , p. 322-323 et fig. 110 .

54. J. Vezin, «La ponctuation... », p. 441.

55. CIFM, t. 22, p. 34 .

56. CIFM, t. 7 , p. 87 et fig. 53 . 


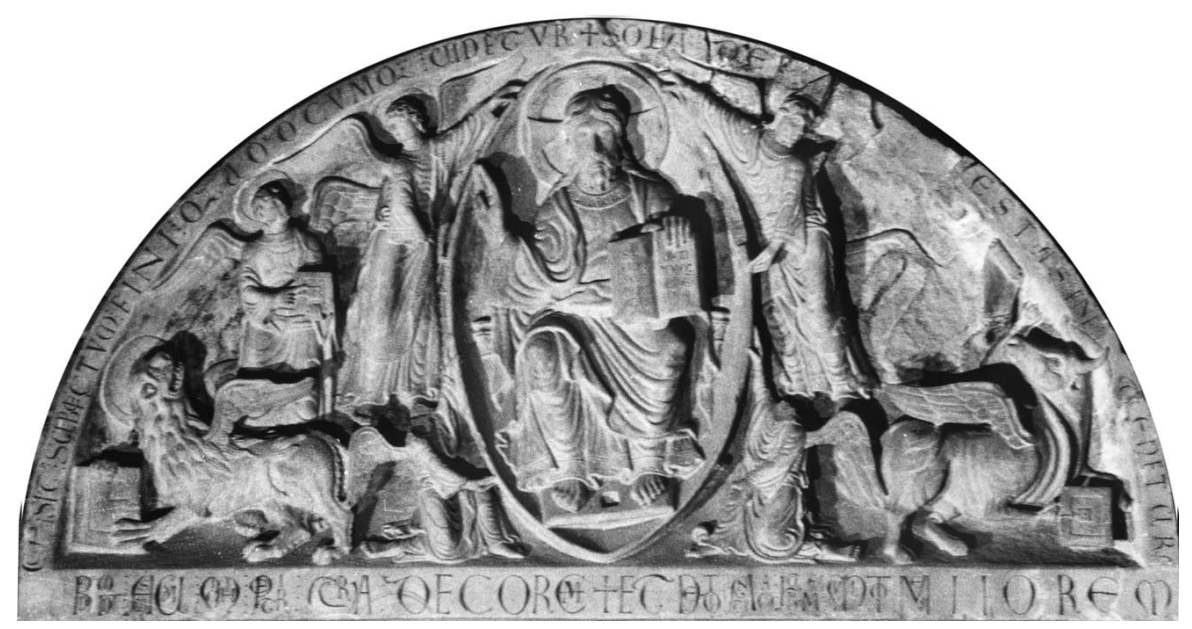

ILL. 8. - Sentence, signature de commanditaire et citation biblique, seconde moitié du XII siècle.

Dijon (Côte-d'Or), musée archéologique, provenant de Saint-Bénigne. Cliché J. Michaud (CESCM/CIFM).

La date est un élément important du texte et les premières ponctuations servent à bien la distinguer. Pour plus de lisibilité, on ajoute un petit $O$ représentant la terminaison de l'ordinal, au-dessus du $M$ du millésime, du ou des $C$ du siècle, de l'année, du jour. Les premiers exemples repérés sont de 1107 à Fécamp (Seine-Maritime), 1116 puis 1142 à Saint-Gilles (Gard), 1148 à Nîmes (Gard) et Arpavon (Drôme) ${ }^{57}$. Cet usage devient ordinaire, mais non constant, à partir de la seconde moitié du XII ${ }^{\mathrm{e}}$ siècle.

La multiplication des liaisons de lettres (conjonctions, enclavements, entrelacements) et des abréviations nuit à la lisibilité mais peut-être volontairement, jeu de lettres réservé au lecteur averti. C'est le cas dans l'inscription du tympan d'un enfeu de Saint-Bénigne de Dijon (ill. 8 ) de la seconde moitié du XII ${ }^{\mathrm{e}}$ siècle ${ }^{58}$ : une surenchère de liaisons intervient dans reddidit amissum mihi Petri cura, suivi d'un élégant et très clair decorem, puis dans et dedit antiqua formam multo, suivi d'un élégant meliorem. Il faudrait étudier l'ensemble des inscriptions pour voir dans quelle mesure le souci général de clarté a pu conduire à limiter les liaisons de lettres.

La très belle inscription des franchises accordées aux hommes de Montélimar par les seigneurs de la ville en $1198{ }^{59}$ offre un autre exemple de recherche de

57. CIFM, t. 22 , p. 250 et fig. 87 ; CIFM, t. 13, p. 70 et fig. 48 , p. 72 et fig. 50 , p. 27 et fig. 12 ; CIFM, t. 16, p. 103 et fig. 62.

58. $C I F M$, t. 20, n 28 , p. 33 et fig. 10.

59. CIFM, t. 16, p. 150-152 et fig. 97. Dans l'inscription de fondation du monastère allemand de Prüfening, en 1119, les lignes paires sont peintes en rouge, de même que les marges des lignes impaires. 
lisibilité : outre la ponctuation régulière, la première lettre de chaque mot, ainsi que l'important mot final, juramus, sont peints en rouge.

Avec cet exemple, nous sommes déjà également dans la recherche d'élégance qui caractérise les inscriptions au XII ${ }^{\mathrm{e}}$ siècle. Celle-ci peut être marquée d'abord par sa présentation dans un cadre qui, s'il est orné, en fait un véritable tableau comme dans l'épitaphe de saint Andéol à Bourg-Saint-Andéol (Ardèche), la charte de franchise de Crest (Drôme) de 1189 ou la dédicace de Saint-Martin de Limeuil (Dordogne) en 1194 ${ }^{60}$. La plupart des épitaphes d'Arles de la fin du $\mathrm{XII}^{\mathrm{e}}$ et du début du XIII ${ }^{\mathrm{e}}$ siècle ${ }^{61}$ sont aussi présentées sous forme de tableau. À la cathédrale d'Elne, dans l'épitaphe d'un archidiacre mort en $1162^{62}$, le texte est précédé d'un encensoir et d'une main divine; et dans celle de Guillaume Jorda ${ }^{63}$, la même finale -is des six vers est mise en commun. À Cheffes (Maine-et-Loire) ${ }^{64}$, la grande inscription sur ardoise de 1167 a aussi un beau cadre orné de volutes. De façon générale, c'est à partir du XII ${ }^{\mathrm{e}}$ siècle que l'on trouve ces cadres entourant l'inscription ${ }^{65}$.

La recherche calligraphique se marque notamment dans la progression très claire des lettres onciales, rondes, au détriment des lettres carrées et tout simplement des formes classiques qui donnent un caractère plus traditionnel, conservateur, solennel. Les lettres carrées, d'origine plus ancienne, décroissent en importance $\mathrm{au} \mathrm{XI}^{\mathrm{e}}$ siècle et au début $\mathrm{du} \mathrm{XII}^{\mathrm{e}}$ siècle mais on peut encore en trouver dans les premières décennies du XIII ${ }^{\mathrm{e}}$ siècle ; les onciales deviennent quant à elles plus nombreuses et plus diversifiées à partir de la fin du XII ${ }^{\mathrm{e}}$ siècle, le tableau variant selon les régions.

Ainsi, à Poitiers en 976-980 (Saint-Hilaire), tous les $C$ et $14 S$ sur 27 sont carrés ; en 1012 (Sainte-Radegonde), tous les $C$ et $G$ sont carrés, mais à la fin du xi ${ }^{\mathrm{e}}$ siècle (Montierneuf, v. 1081, 1096, 1097, 1101), il n'y a plus de lettres carrées 66. En Périgord, en 1117 (Saint-Avit-Senieur), il n'y a pas de lettres carrées et les onciales sont peu nombreuses (3E sur 13); vers 1130 (Saint-Amand-de-Coly), il y a $2 C$ carrés sur 5 et les onciales restent rares; en 1169 (Périgueux), il n'y a qu'un $C$ carré et les onciales sont plus nombreuses ( $M, 6 T$ sur $7,4 E$ sur 12) ; en 1194 (Limeuil), on a un $G$ et $12 C$ carrés sur 34, mais aussi beaucoup d'onciales, les $E$ par exemple étant plus nombreux sous

60. Resp. CIFM, t. 16, fig. 31-32 ; ibid., fig. 65, et R. Favreau, Épigraphie..., p. 40-42, fig. 2 ; CIFM, t. 5, fig. 9.

61. CIFM, t. 14, fig. 42, 43, 47-52 (Saint-Trophime) ; fig. 55 (musée d'Art chrétien) ; fig. 59 (musée Réattu).

62. CIFM, t. 11, fig. 42 .

63. Ibid., fig. 43-44.

64. Xavier Barbier de Montault, Épigraphie du département de Maine-et-Loire, Angers, 1869, p. $18-28$.

65. On trouve tôt de superbes cadres ornés pour des inscriptions latines en Andalousie: épitaphes de 927/966 et de 1002 au musée de Malaga et au musée de Grenade (Arte mozárabe, Tolède, 1975, $\mathrm{n}^{\circ} 4$, p. 15 et pl. II ; n $^{\circ} 6$, p. 16).

66. CIFM, t. 1, fig. 38, 69-71 ; fig. 54, 55, 58-60. 
forme onciale que classique ${ }^{67}$. À Toulouse vers 1050 , tous les $C$ sont carrés et il n'y a pas d'onciale ; avant 1093, on a à nouveau tous les $C$ carrés mais 3 onciales ; en 1167, si les $C$ carrés sont encore présents, il y a désormais beaucoup d'onciales variées ; en 1185, presque toutes les lettres, sauf le $D$, ont la forme onciale ${ }^{68}$. À Narbonne, les lettres carrées sont rares dans la seconde moitié du $\mathrm{XII}^{\mathrm{e}}$ siècle où les onciales se multiplient ${ }^{69}$. À Vienne, tous les $C$ sont encore carrés en 1126 mais ils sont très rares à partir du milieu du siècle ; en 1148, il y a plus de $E$ onciaux que classiques mais en 1152 il y a encore un $E$ oncial isolé ; les formes onciales deviennent nombreuses et diversifiées dans les inscriptions de 1186 et de 1188 70. En Roussillon, il n’y a aucune onciale en 1019-1020 ; encore $8 C$ carrés sur 16 en 1144 mais les onciales sont dès lors présentes en force $^{71}$; à Elne, les $E$ onciaux sont au nombre de 8 sur 27 en 1144, 8 sur 23 en 1162, 10 pour 18 en 1186, 6 pour 9 en 1201 et on compte $3 M$ onciaux sur 10 en 1144, 5 sur 9 en 1162, 1 sur 5 en 1186, 5 sur 7 en $1201{ }^{72}$.

Il faudrait en outre étudier l'évolution des différentes lettres onciales, particulièrement marquée pour les $M$ et les $T$, en notant que, dans la même inscription, on a fréquemment deux ou trois formes onciales nettement différentes pour les $M$, deux types bien différenciés pour les $T$. Dresser des tableaux de formes de lettres peut s'avérer fructueux, mais que de difficultés à éviter dans la mesure où il faut tenir compte du support, de la région, du lieu de production! Surtout, il faut éviter de partir d'une lettre isolée dans un tableau pour en tirer une datation.

Les onciales qui peu à peu envahissent l'inscription sont de plus en plus élégantes : on rejoint ici l'élément le plus évident de cette recherche nouvelle d'une belle écriture, le développement de lettres ornées ou fleuries, c'est-à-dire de lettres où des enjolivements sont ajoutés à la morphologie ordinaire. Une lettre ornée s'observe sur la seconde plaque de l'autel majeur de la cathédrale d'Elne commémorant la fondation de cet autel en $1069^{73}$, mais il faudrait peut-être revoir la datation de cette inscription, premier témoin, avec beaucoup d'avance également, de l'apparition de lettres perlées et à étranglement. Plus assurées sont les lettres fleuries des inscriptions datées de Vienne (Isère) en 1126, Lesterps (Charente) vers 1140 et Elne (Pyrénées-Orientales) en 1144 ${ }^{74}$, Vienne en 1148 et 1152, Bures-en-Bray (Seine-Maritime) en 1168 (ill.9), Vienne en 1170, Toulouse (Haute-Garonne) en 1173, Elne en 1201, 1209, 1213

67. CIFM, t. 5 , fig. 41 ; fig. 39 ; fig. 15 ; fig. 9.

68. CIFM, t. 7, fig. $44 ;$ p. 41 , dessin ; fig. $56 ; 57$.

69. $C I F M$, t. 12 , fig. 36,39 .

70. CIFM, t. 15, fig. $41-43 ; 64-65 ; 47-51 ; 56$ et 57 .

71. CIFM, t. 11, fig. 106 ; fig. 41.

72. CIFM, t. 11, fig. $41,42,43-44,47$.

73. CIFM, t. 11, fig. 38 .

74. CIFM, t. 15, fig. 42 ; t. 3, fig. $26-28$; t. 11, fig. 41, enjolivement à l'intérieur d'un $O$. On a de semblables enjolivements dans deux lettres d'une inscription du musée de Poitiers, dont il faudrait peut-être revoir la datation proposée, la seconde moitié du xI ${ }^{\mathrm{e}}$ siècle (CIFM, t. $1, \mathrm{n}^{\circ} 86$ et fig. 47). 


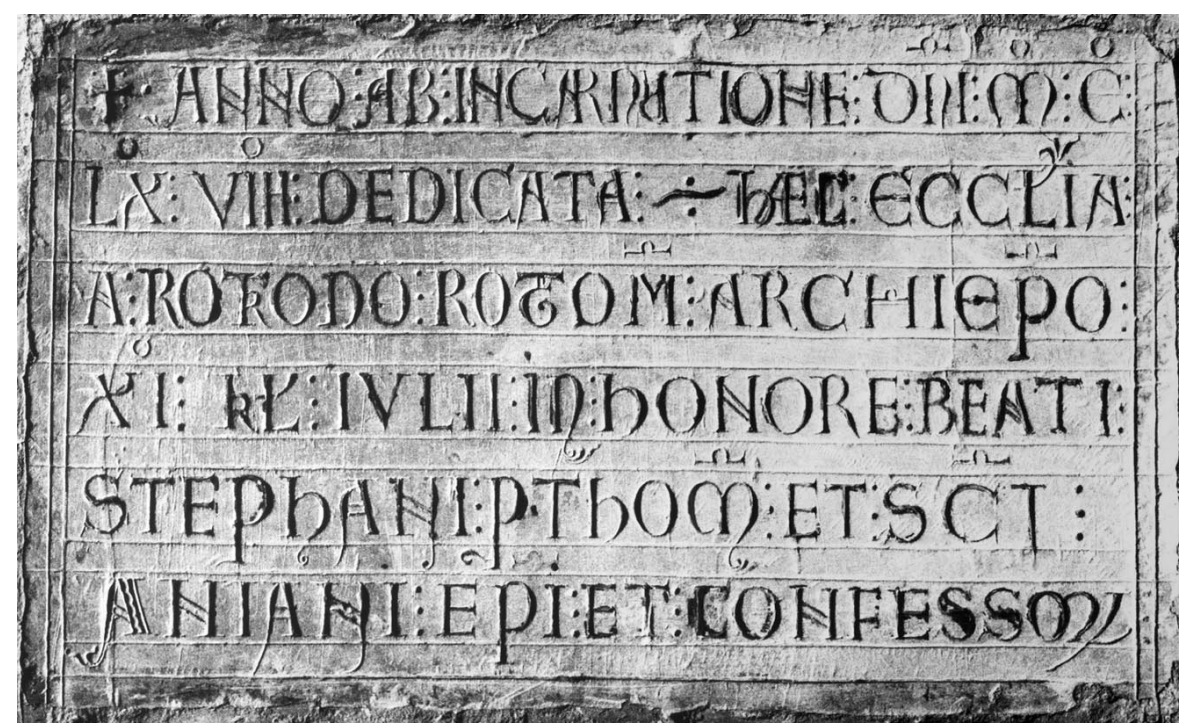

ILL. 9. - Dédicace de l'église, 1168. Bures-en-Bray (Seine-Maritime), église, dernière travée du chœur. Cliché J.-P. Brouard (СЕsCM/CIFM).

ou encore Bourg-Saint-Andéol (Ardèche) en 1207 (ill. 10) ${ }^{75}$. L'enjolivement des lettres se pratique principalement à partir du deuxième quart du XII ${ }^{\mathrm{e}}$ siècle et se poursuivra sur une bonne partie du XIII ${ }^{\mathrm{e}}$ siècle ${ }^{76}$.

Le redoublement des traits participe aussi à la recherche de qualité visuelle. Dans les inscriptions ici considérées, le premier exemple daté serait celui de la bague du bâton pastoral d'Adémar, évêque d'Angoulême $(† 1101){ }^{77}$, où l'on a marqué, ce qui est exceptionnel, le décès du prélat : toutes les lettres y sont à traits redoublés. De façon plus ordinaire, ce sont surtout les $A$ et les $N$ qui seront touchés par ce genre d'ornement. Une lettre redoublée s'observe dans une inscription de 1117 à Saint-Avit-Sénieur (Dordogne) ${ }^{78}$. Les autres exemples interviennent, cette fois en nombre, à partir de la seconde moitié du siècle, dans toute la France du sud. On trouve aussi des redoublements de traits à Bures-en-Bray, 1168, et à Osmoy-Saint-Valery, 1170, en Normandie ${ }^{79}$.

75. $C I F M$, t. 15 , fig. 43,48 ; t. 22 , fig. 76 ; t. 15 , fig. 54 ; t. 7 , fig. 54 ; t. 11 , fig. $47,51,52$; t. 16 , fig. 37.

76. Il y a donc un décalage chronologique par rapport à ce que Jacques Stiennon a observé dans l'écriture diplomatique du diocèse de Liège, et plus généralement en pays mosan et rhénan, du dernier quart du XI ${ }^{\mathrm{e}}$ siècle à la première moitié du XIII ${ }^{\mathrm{e}}$ siècle : J. Stiennon, L'écriture diplomatique dans le diocèse de Liège du XI ${ }^{e}$ au milieu du XIII siècle, reflet d'une civilisation, Paris, 1960 (Bibliothèque de la faculté de philosophie et lettres de l'Université de Liège, gr. in-8º, 5) ; voir aussi id., La paléographie du Moyen Age, Paris, 1973, p. 104.

77. CIFM, t. $3, \mathrm{n}^{\circ} 18$ bis, p. 128 et fig. 16 bis-16 ter.

78. CIFM, t. 5, fig. 41.

79. CIFM, t. 22, fig. 76 et 98 . 


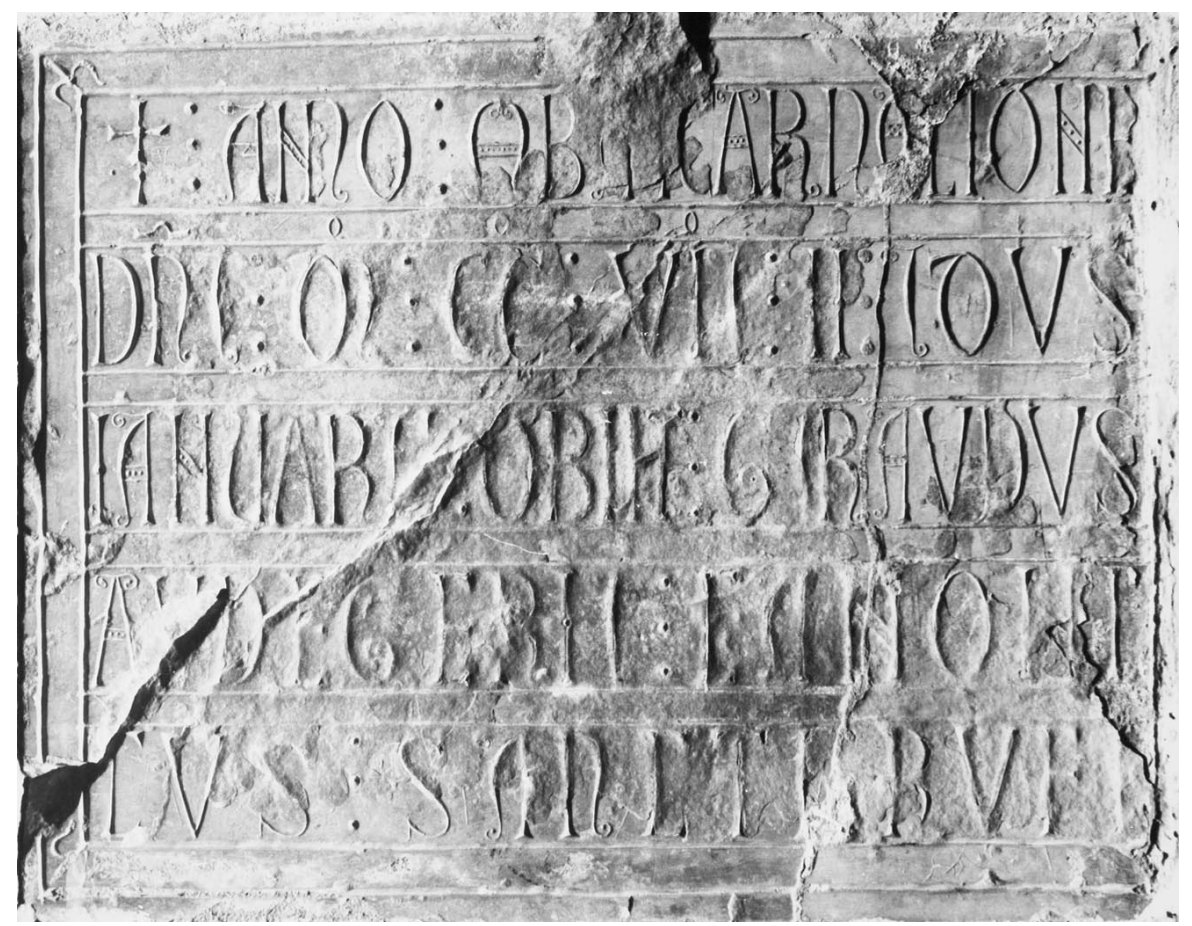

ILL. 10. - Inscription funéraire de Giraud Audegier, 1207. Bourg-Saint-Andéol (Ardèche), ancien couvent Saint-Ruf. Cliché J. Michaud (СЕscm/CIFM).

Les tildes d'abréviation tracés avec un renflement médian peuvent être joints à cette recherche du trait orné. On trouve un tilde à renflement dans l'épitaphe de l'abbé Aymar (†963) au musée Ochier de Cluny ${ }^{80}$; l'épitaphe est nettement postérieure à la mort de l'abbé, et vraisemblablement de la seconde moitié du $\mathrm{XI}^{\mathrm{e}}$ siècle. Un exemple plus assuré est celui de l'inscription de consécration d'un autel de Saint-Jean-de-Montierneuf à Poitiers par Germond, archevêque de Vienne (1077-1081) ${ }^{81}$. Le tilde à renflement devient dès lors de plus en plus courant tout au long du XII ${ }^{\mathrm{e}}$ siècle. On trouvera aussi des renflements dans la traverse du $H$, la barre oblique du $N$. Cette particularité s'observe notamment à Vienne, où l'on a aussi un renflement médian dans la haste du $I$ et du $T$.

Les lettres à étranglement, qui introduisent un pincement dans une courbe, semblent une particularité de la France du sud-ouest et du sud-est, même si l'on peut noter deux exceptions: la signature d'un artiste dans l'abbatiale de

80. CIFM, t. 19, fig. 108.

81. CIFM, t. $1, \mathrm{n}^{\circ} 73$, fig. 55 . 
Tournus (Saône-et-Loire), où le $O$ présente un double étranglement, peu accentué mais net ${ }^{82}$, et l'épitaphe de l'abbé Joscerand à Saint-Benoît-sur-Loire (1096), où trois $T$ auraient une forme onciale avec étranglement dans la courbe d'après le fac-similé donné par dom Chazal ${ }^{83}$. À part l'inscription d'Elne de 1069 déjà citée, tous les exemples se situent aux XII ${ }^{\mathrm{e}}$ et XIII ${ }^{\mathrm{e}}$ siècles, dans un étroit parallélisme avec les lettres ornées. Les inscriptions de 1173 et 1174 à Toulouse, 1162 ou 1209 à Elne, 1211 à Narbonne et 1239 à Vienne ${ }^{84}$ en offrent des exemples particulièrement riches ${ }^{85}$.

Moins répandues, les lettres perlées sont un autre élément caractéristique de la même recherche intentionnellement calligraphique. Pour un ou deux exemples au xI $^{\mathrm{e}}$ siècle (Limoges, Elne ${ }^{86}$ ), elles se rencontrent régulièrement, sinon souvent, à partir de la seconde moitié du XII ${ }^{\mathrm{e}}$ et au XIII ${ }^{\mathrm{e}}$ siècle.

Le souci d'une plus grande facilité de lecture, notamment par l'espacement des mots et par la ponctuation, a sa place dans l'élargissement social de la culture écrite aux $\mathrm{XI}^{\mathrm{e}}-\mathrm{XII}^{\mathrm{e}}$ siècles, du fait du développement des écoles et de l'accession plus large des laïcs à une activité jusque-là essentiellement réservée aux clercs, aspects d'une civilisation globalement plus soucieuse des usages de l'écrit : renaissance du droit romain au $\mathrm{XII}^{\mathrm{e}}$ siècle, apparition de besoins nouveaux avec le développement du commerce, pratique plus individuelle de la piété où la lecture privée gagne en importance. La recherche décorative, qui se manifeste surtout à compter de la seconde moitié du XII ${ }^{\mathrm{e}}$ siècle, correspond à un changement de goût, à un nouveau dynamisme qui se substitue à l'équilibre de l'âge roman. Alors que l'épigraphie a généralement, par vocation, un caractère conservateur, l'évolution graphique des inscriptions est étroitement liée aux changements sociaux si manifestes à la fin du Moyen Âge.

\section{De la majuscule « Onciale » ̀̀ la minuscule gothique $\left(\mathrm{XIII}^{\mathrm{e}}-\mathrm{XV}^{\mathrm{e}}\right.$ SIÈCLE $)$.}

Les changements de l'écriture épigraphique à partir du XIII ${ }^{\mathrm{e}}$ siècle reposent à la fois sur une évolution lente et profonde des formes, entamée dès le XII ${ }^{\mathrm{e}}$ siècle avec le développement de l'onciale, et sur une série d'événements qui finiront

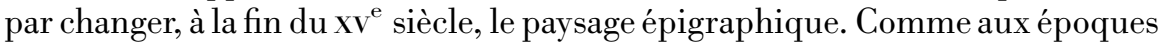
précédentes, les changements dans les inscriptions ne peuvent être séparés ni des évolutions que connaît au même moment l'écriture dans les manuscrits, ni,

82. CIFM, t. 19, fig. 161-162.

83. Boucher de Molandon, "Inscriptions tumulaires... », pl. IV, fig. 3.

84. CIFM, t. 7, fig. 54, 57 ; t. 11, fig. 48, 51 ; t. 12, fig. 41 ; t. 15, fig. 79-80.

85. Il faudrait d'ailleurs étendre la recherche à l'Italie du sud et à l'Espagne, où l'on retrouve les mêmes formes, notamment en Catalogne à Castellar del Vallés, Gérone, Vic, Tahull, Tortosa, en Aragon à San Pedro el Viejo de Huesca, en Castille à León, Burgos, Salamanque, et en Galice (inscription provenant de Compostelle conservée au musée de Madrid).

86. CIFM, t. 4 , fig. 56 ; t. 11, fig. 37-38. 
de façon plus large, des transformations de la culture écrite médiévale dans son ensemble. Les différences régionales d'écriture persistent pour la fin du Moyen Âge ; on constate cependant que celles-ci tendent à s'atténuer, la paléographie des inscriptions présentant un aspect beaucoup plus uniforme. Si différence il y a, celle-ci se situe beaucoup plus, d'une région à l'autre, dans le décalage chronologique de l'évolution des formes, et non plus dans le caractère proprement « régional » des écritures.

Le souci de clarté et la recherche d'élégance, évoqués pour le Moyen Âge central, ne disparaissent pas au cours des $\mathrm{XIII}^{\mathrm{e}}-\mathrm{XV}^{\mathrm{e}}$ siècles. Les implications sont toutefois différentes sur l'évolution de la forme des lettres. Les critères de lisibilité et d'élégance se colorent, à partir du XIII ${ }^{\mathrm{e}}$ siècle, des caractéristiques de l'esthétique gothique, au même titre que dans l'écriture des livres. Les correspondances formelles mises au jour par Erwin Panofsky entre modes de pensée, structures architecturales et éléments paléographiques se retrouvent ainsi, pour la plupart, dans les inscriptions ${ }^{87}$. On tend alors peu à peu vers l'homogénéisation de la graphie, vers l'abandon des formes complexes et ornées et vers l'unité du texte.

Le XIII $^{\mathrm{e}}$ siècle marque le triomphe dans les inscriptions d'une majuscule " onciale " ${ }^{88}$, utilisée dès le XI $^{\mathrm{e}}$ et surtout le XII ${ }^{\mathrm{e}}$ siècle, et qui s'impose nettement sur l'ensemble du territoire français dans la première moitié du siècle suivant. Les inscriptions datées autour de 1200 présentent encore un mélange entre les lignes courbes des onciales et les traits droits des lettres carrées ou des capitales. L'épitaphe de l'évêque Arnaud IV (mort en 1201), conservée dans la cathédrale d'Elne 89 (Pyrénées-Orientales), présente des formes onciales et capitales pour les lettres $A, E, I, L, M, N, P, T$ et $U$. Trente ans plus tard, l'inscription peinte dans l'église de Saint-Christophe-du-Jambet (Sarthe) ${ }^{90}$ offre encore une grande proportion de lignes droites, même si les onciales ont fait leur apparition avec les lettres $C, E, M$ et $T$. En France, on peut dire que vers 1250, les onciales ont pratiquement fait disparaître les capitales, au nord comme au sud. Dans l'épitaphe d'Édeline la Charretière, gravée en 1246 et conservée à Sens ${ }^{91}$, toutes les lettres présentent une forme onciale. La lettre $E$ paraît constituer un bon témoin de la généralisation du recours à l'onciale. En effet, le $E$ carré ou capital n'apparaît pratiquement plus dans les inscriptions françaises datées à partir de 1240-1250. Il apparaissait quatre fois

87. Erwin Panofsky, Architecture gothique et pensée scolastique, trad. fr., Paris, $1974\left[1^{\mathrm{re}}\right.$ éd. 1967] (Le sens commun), p. 79.

88. Rappelons que la tradition épigraphique appelle onciales toutes les lettres qui se distinguent par leur rondeur de la forme classique, et non seulement celles qui sont issues de l'écriture « onciale» selon la nomenclature des paléographes. La "majuscule onciale» désigne ici ce que d'autres appellent "majuscule gothique " par opposition à la "minuscule gothique " des XIV $\mathrm{Xv}^{\mathrm{e}}$ siècles, dont il sera question plus bas.

89. CIFM, t. $11, \mathrm{n}^{\circ} 51$, p. 63.

90. Inscription citée par Marcel Deyres, Maine roman, La Pierre-qui-Vire, 1985, p. 52.

91. CIFM, t. 21, no 165, p. 182. 


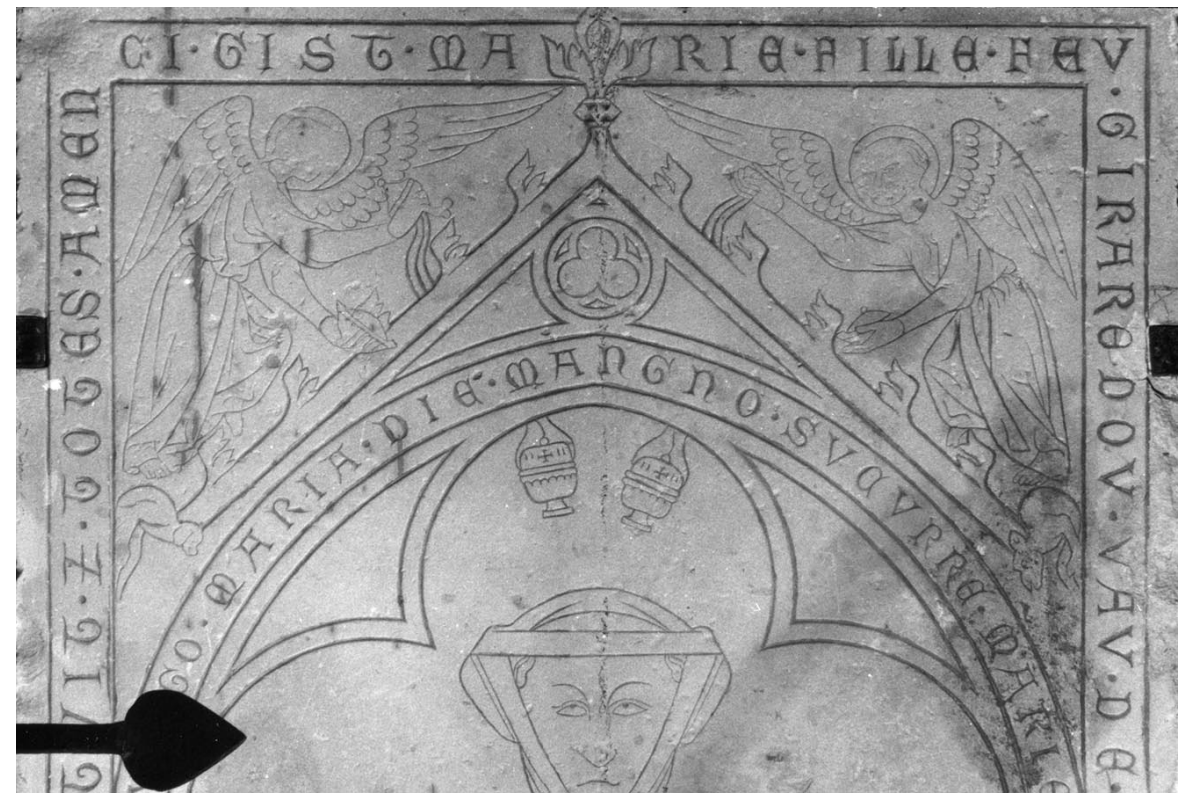

ILt. 11. — Dalle tumulaire de Marie, 1299. Villeroy (Yonne), église, nef. Cliché J.-P. Brouard (CESCM/CIFM).

dans une inscription datée de 1239 , conservée à Vienne ${ }^{92}$, mais il est complètement absent des inscriptions du sud-est de la France à partir du milieu du $\mathrm{XIII}^{\mathrm{e}}$ siècle, ou presque : toute généralisation de cette sorte est excessive et dépend essentiellement d'un état partiel de la documentation; ainsi, dans l'épitaphe de Pons, gravée dans la tour du Diable de Villefranche-de-Conflent, en $1286^{93}$, on retrouve des lettres carrées, aux formes très droites qui peuvent, pour certaines, rappeler l'écriture des $\mathrm{IX}^{\mathrm{e}}-\mathrm{x}^{\mathrm{e}}$ siècles. De tels exemples restent toutefois très marginaux. Au total, à la fin du XIII ${ }^{\mathrm{e}}$ et au début du XIV $^{\mathrm{e}}$ siècle, les inscriptions atteignent, au nord comme au sud de la France, une certaine perfection onciale, avec des inscriptions très homogènes, composées de lettres aux lignes courbes soignées et régulières. On peut comparer, de ce point de vue, la dalle tumulaire de Marie (ill. 11), morte en 1299, conservée à Villeroy (Yonne), et l'épitaphe de Pierre Roger, mort en 1307, conservée à Unzent (Ariège) ${ }^{94}$. Les deux textes, fort éloignés géographiquement l'un de l'autre, présentent pourtant des formes très similaires. L'adoption par les inscriptions de la lettre onciale est donc pratiquement totale vers 1300 et le restera jusqu'à la fin du XIV siècle.

92. $C I F M$, t. $15, \mathrm{n}^{\circ}$ 104, p. 101-102.

93. $C I F M$, t. $11, \mathrm{n}^{\circ} 138$, p. 143.

94. $C I F M$, t. $21, \mathrm{n}^{\circ} 231$, p. 250 et $C I F M$, t. 8, nº 8 , p. 19. 
L'harmonisation des formes autour de l'onciale est facilitée par la réduction progressive du nombre des formes pour chaque lettre. Ce souci correspond à une recherche d'unité pour le texte que l'on retrouve également dans l'écriture manuscrite ${ }^{95}$. Dans l'inscription de la cathédrale d'Elne de 1201 déjà mentionnée ici, on compte seulement 20 lettres pour composer le texte, mais plus de 70 formes différentes : 4 types de $A, 3$ types de $C, 4$ types de $M, 4$ types de $N, 5$ types de $T$, etc. En 1239, à la cathédrale Saint-Maurice de Vienne, l'épitaphe de Charles et Colombe ${ }^{96}$ présente encore 4 types de $A, 5$ types de $E, 4$ types de $R$, 4 types de $T, 4$ types de $U$ et 3 types de $X$. Dans l'inscription de la châsse de Saint-Taurin d'Evreux ${ }^{97}$, réalisée entre 1240 et 1255, on a en moyenne 4 formes par lettre. À partir du milieu du XIII ${ }^{\mathrm{e}}$ siècle, le rapport moyen du nombre de formes par lettre diminue fortement. En 1268 par exemple, dans l'épitaphe de Guillaume Porcell ${ }^{98}$ conservée à Pia (Pyrénées-Orientales), on ne compte plus qu'une moyenne de 2 formes par lettre avec, fait exceptionnel, une surreprésentation de la rare lettre $G$ (sous 3 formes). En 1298, à Saint-Germain-enBrionnais (Saône-et-Loire), l'épitaphe de Sibylle de Luzy 99 ne compte plus qu'une seule forme pour la plupart des lettres (ill. 12). En 1313, une des épitaphes de l'abbaye de Lérins à Cannes ${ }^{100}$ (Alpes-Maritimes) ne présente plus qu'une forme par lettre, à l'exception du $M$. Cela devient au XIv ${ }^{\mathrm{e}}$ siècle une règle générale, qui se maintiendra au $\mathbf{X v}^{\mathrm{e}}$ pour celles des inscriptions qui resteront fidèles aux majuscules onciales. Cette uniformisation de l'écriture épigraphique autour d'un modèle unique de lettres ne constitue pas en soi une nouveauté. Même si elle ne procède pas d'une volonté politique d'uniformiser l'écriture, la recherche d'une unité formelle pour le texte n'est pas sans rappeler ce que l'on peut trouver dans les inscriptions tracées avec les capitales choisies par la réforme carolingienne. Les critères esthétiques ont changé mais ils ne suffisent pas à expliquer ce souci d'uniformisation. Sans doute faudrait-il en rechercher les causes dans la pratique de l'écriture manuscrite elle-même et dans sa capacité à former des goûts, des modes, des canons.

La majuscule onciale se caractérise par le recours à la ligne courbe. La morphologie de la lettre est arrondie, ses proportions sont généralement carrées, parfois oblongues, sans jamais dépasser un rapport d'un sur deux. Au cours des $\mathrm{XIII}^{\mathrm{e}}-\mathrm{XV}^{\mathrm{e}}$ siècles, ces données générales définissant la forme onciale vont connaître des transformations substantielles. On assiste d'abord à une fermeture progressive des lettres. Les lapicides insistent sur la courbure de telle sorte que les traits finissent par se distribuer autour du centre du signe. Les extrémités supérieure et inférieure des lettres rondes, telles que le $E$, le $C$, le $G$

95. P. Saenger, « Coupure et séparation... », p. 455.

96. CIFM, t. $15, \mathrm{n}^{\circ} 28$, p. $31-32$.

97. CIFM, t. $22, \mathrm{n}^{\circ} 85$, p. 144.

98. CIFM t. 11, $\mathrm{n}^{\circ} 109$, p. 106.

99. $C I F M$, t. $19, \mathrm{n}^{\circ} 72$, p. 128.

100. Inscription mentionnée et reproduite dans Henri Moris, L'abbaye de Lérins : histoire et monuments, Paris, 1909, p. 388-389. 


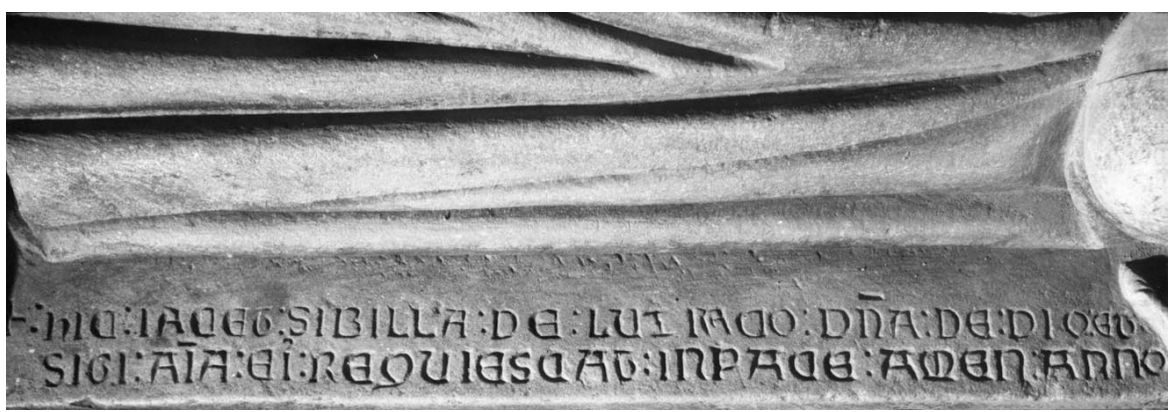

ILL. 12. - Inscription funéraire de Sibylle de Lusy, 1298. Saint-Germain-en-Brionnais (Saône-et-

Loire), église, collatéral nord, $2^{\mathrm{e}}$ travée à partir de l'ouest. Cliché J. Michaud (CEscm/CIFM).

ou le $T$, se rejoignent pour former ce qui ressemble, dans la seconde moitié du XIII ${ }^{\mathrm{e}}$ siècle, à un cercle. C'est ce que l'on remarque par exemple dans l'épitaphe d'Atenulfe, datée de 1248 et conservée dans l'ancienne commanderie SaintJean-des-Prés de Montbrison (Loire) ${ }^{101}$. Dans cette belle réalisation, les lignes courbes du $T$ oncial forment pratiquement un cercle parfait que vient chapeauter une ligne droite. Dans la même inscription, les deux jambes extérieures du $M$ rejoignent celle du milieu, fermant ainsi complètement la lettre. De même, le $C$, le $E$, le $H$ et le $S$ sont pratiquement fermés.

Cette systématisation de la fermeture des signes est d'autant plus évidente qu'elle affecte des lettres plus éloignées d'un tracé circulaire, telles que $S, A, F$ ou encore $U$. À partir de 1250 , ces signes offrent de plus en plus souvent une forme fermée, soit par le rapprochement des éléments morphologiques, soit par l'adjonction d'un trait supplémentaire. En 1300, dans une inscription de Saint-Bertrand-de-Comminges (Haute-Garonne) ${ }^{102}$, cette transformation arrive à une sorte de paroxysme: des traits additionnels viennent fermer pratiquement tous les signes, qu'il s'agisse des $E$, des $C$ ou des $U$, comme à Sens, mais aussi le $K$, le $H$, les $N$, le $P$, le $R$ et le $S$ (ill. 13). Ces lignes de fermeture prennent parfois une importance telle qu'elles débordent des proportions générales. Dans l'inscription de Villeroy (1299), le $C$ est par exemple fermé d'une ligne deux fois plus haute que la lettre; les barres horizontales du $F$ sont fermées par un trait qui a la même hauteur que la lettre elle-même, etc. Une lettre normalement composée de 2 traits, comme le $L$ ou le $T$, se verra complétée par des lignes verticales unissant les barres horizontales avec le sommet ou le pied de la lettre. Dans d'autres cas, pour les $A$ et les $R$ notamment, la fermeture intervient par le rapprochement puis la réunion des traits verticaux, comme dans l'inscription de Saint-Bertrand-de-Comminges.

L'utilisation exclusive de l'onciale et la fermeture des lettres ont une double conséquence sur l'évolution paléographique des inscriptions, surtout à partir

101. $C I F M$, t. $18, \mathrm{n}^{\mathrm{o}} 22$, p. 75 .

102. CIFM, t. 8, n 33, p. 68. Épitaphe de Raimond de Magrens (28 octobre 1300). 


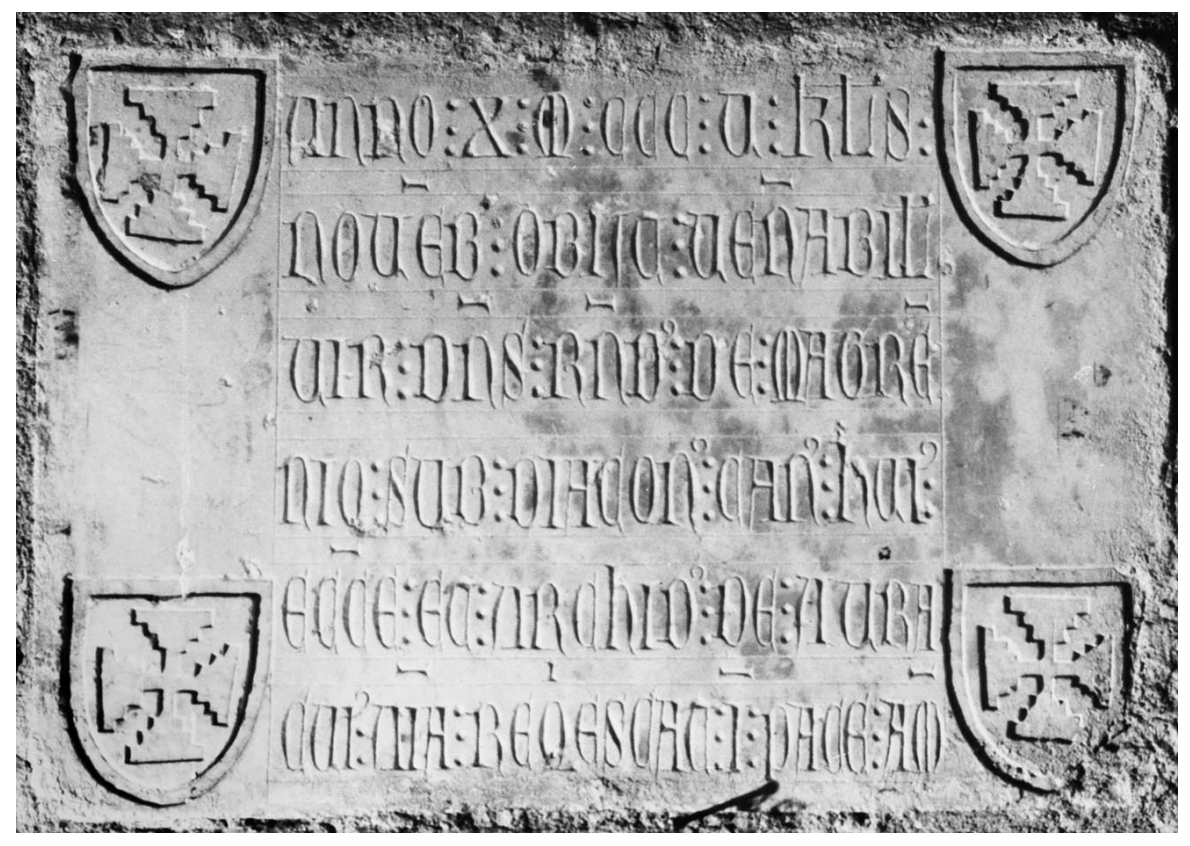

ILL. 13. - Inscription funéraire de Raimond de Magrens, 1300. Saint-Bertrand-de-Comminges

(Haute-Garonne), cathédrale, cloître, galerie nord. Cliché J. Michaud (сEscm/CIFM).

de la seconde moitié du XIII ${ }^{\mathrm{e}}$ siècle. On assiste d'abord à un étrécissement du module des lettres. Si les époques antérieures se caractérisaient par un rapport modulaire (c'est-à-dire la proportion entre largeur et hauteur des lettres) de 1/1 ou 1/2, la fin du Moyen Âge voit cette relation se réduire encore. Le XIII ${ }^{\mathrm{e}}$ siècle passe ainsi d'une largeur deux fois moins grande que la hauteur au minimum, comme dans l'inscription d'Elne, à une relation de $1 / 3$ pour certaines lettres, comme dans celle de Montbrison. À partir de 1270 environ, le rapport modulaire semble se stabiliser autour de 1/4, et on ne trouve que peu d'exemples revenant à un rapport plus important. Une telle compression latérale va de pair avec une augmentation de la hauteur des signes. Comme dans l'écriture manuscrite, fait déjà signalé par E. Panofsky, c'est le triomphe de la verticalité, l'étirement en hauteur, qui domine l'ensemble de l'évolution de la paléographie des inscriptions. Un deuxième corollaire est le recours de plus en plus systématique à l'alternance entre les pleins et les déliés. Dans le monde manuscrit, cette différence de graisse est une conséquence technique des instruments utilisés pour écrire. Dans le cas des inscriptions, la gravure en creux de la pierre grâce à un outil tranchant ne justifie en rien une telle variation dans le poids de la ligne : elle résulte donc plutôt d'une imitation des caractéristiques de l'écriture manuscrite. De technique, l'emploi des pleins et des déliés devient purement stylistique dans les inscriptions et constitue un aspect de l'onciale dans sa 
perfection. Là où l'on trouvait, dans l'écriture manuscrite, un plein, c'est-à-dire un trait plus épais, on trouve dans l'inscription un trait plus fort, plus profondément incisé dans le support ou simplement plus large. Au cours du XIII ${ }^{\mathrm{e}}$ siècle, le contraste entre plein et délié ne fait que s'accroître, tant et si bien que le trait fin du délié disparaît peu à peu de la graphie, comme dans l'épitaphe d'Atenulfe. Dans la lettre $A$, par exemple, la courbe de la première barre verticale est exagérément large alors que la terminaison de cette même ligne se perd dans le support tant elle est fine. La courbe de la lettre $E$ est particulièrement renforcée alors que la ligne verticale qui ferme la lettre se distingue à peine. Dans les inscriptions du cloître de Saint-Bertrand-de-Comminges, datées pour la plupart des dernières années du XIII ${ }^{\mathrm{e}}$ siècle ou des premières $\mathrm{du}$ $\mathrm{XIV}^{\mathrm{e}}$, les traits verticaux des lettres sont traités en plein et les autres en délié ; l'image renvoyée par le texte tend de plus en plus à montrer une succession de lignes verticales fortes, et de moins en moins de traits fins complémentaires, horizontaux ou transversaux. C'est également l'impression qui se dégage de l'inscription conservée dans la ferme poitevine de la Grande Vacherie : les traits fins ont disparu et les traits pleins se développent. La lettre onciale $N$ se compose uniquement de deux traits forts, verticaux et parallèles, le $O$ de deux traits courbes et larges, etc. Au terme de toutes ces transformations, l'écriture onciale arrive à une remise en question de sa définition.

L'évolution formelle entamée dans les premières années du XIII ${ }^{\mathrm{e}}$ siècle avec la systématisation de l'onciale trouve une sorte d'accomplissement dans l'apparition de la minuscule gothique dans les inscriptions. La succession de traits verticaux pleins que l'on avait relevée dans les textes de Saint-Bertrand-deComminges n'est pas sans rappeler, d'une certaine manière, l'aspect de la minuscule gothique. Celle-ci se compose en effet, pour de nombreuses lettres, d'une série de traits verticaux (trois pour le $M$, deux pour le $N$, un pour le $L$, etc.) et, comme pour les déliés des majuscules onciales, les traits horizontaux ou transversaux de la minuscule gothique tendent à disparaître de la surface du support. Cela ne signifie évidemment pas qu'il faille établir une filiation de structure entre ces deux types, qui ont chacun sa généalogie ; en revanche, il faut y voir une continuité esthétique très nette. L'apparition de la minuscule des livres dans le monde épigraphique constitue une véritable nouveauté, qu'il ne faut pas confondre avec le recours spontané à une écriture usuelle. La minuscule épigraphique obéit toujours à une certaine solennité et parfois à une recherche calligraphique plus poussée. Il est encore difficile de fixer son apparition dans les inscriptions mais il semblerait que les premiers témoins datent des années 1350. Si le développement est surtout sensible au $\mathbf{X v}^{\mathrm{e}}$ siècle, il n'entraînera jamais la disparition de l'usage de la majuscule, d'abord onciale ou gothique, puis humanistique, dans les textes épigraphiques ${ }^{103}$. En revanche, l'emploi de la minuscule gothique a des implications fondamentales pour la fonction et la lecture des inscriptions. Si leur capacité à transmettre un

103. Ottavio Banti, «Dall'epigrafica romanica alla preumanistica : la scrittura epigrafica dal XII alla fine del xv secolo a Pisa », dans Scrittura e civiltà, t. 24, 2000, p. 61-102, à la p. 97. 
message ne diminue pas — c'est une perception tout à fait anachronique qui fait dire que la minuscule gothique est peu lisible - les inscriptions transcrites en minuscule ne peuvent plus en revanche se contenter d'être aperçues par le lecteur ; le rapport entre texte et récepteur est alors plus intime. C'est sans doute la raison pour laquelle la plupart des inscriptions publicitaires restent encore tracées en majuscules (y compris d'ailleurs dans les manuscrits, pour les titres, les principales rubriques, etc.) ${ }^{104}$.

Les caractéristiques formelles de la minuscule gothique dans les inscriptions ne sont pas fondamentalement différentes de ce que l'on trouve dans les textes manuscrits. Composée majoritairement de traits verticaux inclinés dans la partie supérieure, elle présente un tracé simple et homogène ainsi qu'un module plus petit qui revient à des rapports de $1 / 2$, voire de $1 / 1$ dans certains cas. Les hastes des lettres hautes (comme le $t$, le $p$, le $b$, le $h$ ou le $d$ ) sont souvent bifides et les lettres basses peuvent être complétées par un trait fin placé sous le tracé originel. Pas plus que dans les majuscules onciales des XIII ${ }^{\mathrm{e}}-\mathrm{XIV}^{\mathrm{e}}$ siècles, on ne rencontre de véritables tracés ornementaux dans la minuscule. Le souci calligraphique se note plutôt dans le recours à la majuscule initiale, comme l'avait remarqué $\mathrm{O}$. Banti, et de façon générale dans la recherche d'homogénéité ${ }^{105}$. La principale conséquence de l'emploi de la minuscule gothique réside dans le rapprochement de plus en plus évident entre les formes épigraphiques et manuscrites de l'écriture. La graphie gothique s'impose véritablement au cours $\mathrm{du} \mathrm{xv}^{\mathrm{e}}$ siècle, indépendamment du support de l'écriture, y compris pour les majuscules. Le retour des proportions carrées et le maintien des majuscules pour certains textes (ou certaines parties) n'ont pas inspiré dans les inscriptions françaises, à la fin du Moyen Âge, de recours à la capitale romaine des humanistes. Il faut attendre l'extrême fin $\mathrm{du} \mathbf{X v}^{\mathrm{e}}$ ou surtout le $\mathrm{XVI}^{\mathrm{e}}$ siècle et la Renaissance française, pour assister au retour de longs textes transcrits en belles capitales parfaitement droites. Il y a là une différence avec ce que l'on rencontre au même moment non seulement en Italie mais également en Espagne ou en Allemagne. L'évolution graphique, dans les inscriptions des $\mathrm{XIII}^{\mathrm{e}}-\mathrm{XV}^{\mathrm{e}}$ siècles, ne peut être caractérisée, pour la France, par de brusques changements formels mais plutôt par l'adoption progressive de types originaux ; cette évolution se fait conformément à l'évolution paléographique des livres mais témoigne dans le même temps d'adaptations fonctionnelles et formelles propres aux inscriptions.

À la fin du Moyen Âge, l'esthétique de l'écriture épigraphique ne repose plus sur l'ornementation des signes. Les lettres fleuries, perlées ou redoublées disparaissent progressivement des inscriptions à partir de la seconde moitié du XIII $^{\text {e }}$ siècle. On rencontre encore des points ornant les hastes des $M$ et des $I$ dans l'épitaphe d'Adémar de Melet au musée de Périgueux en $1263^{106}$. Les traits

104. Armando Petrucci, Jeux de lettres : formes et usages de l'inscription en Italie (XI'$X X^{e}$ s.), trad. fr., Paris, 1995, p. 81-83.

105. O. Banti, « Dall'epigrafica romanica... », p. 95.

106. CIFM, t. $5, \mathrm{n}^{\circ} 46$, p. 58 . 
transversaux de certains $N$ ou $M$ de l'épitaphe de maître Guillaume à SaintAndré-le-Bas de Vienne ${ }^{107}$, gravée en 1238, sont encore redoublés. Dans les deux cas, les ornements graphiques se limitent cependant à quelques lettres isolées, et ce sera la tendance générale jusqu'à la fin du Moyen Âge ${ }^{108}$. Cet abandon progressif des lignes complémentaires ornant la lettre est une conséquence de l'évolution esthétique générale, avec l'adoption de types uniformisés et de lettres régulières. Il s'agit également d'une conséquence technique. La fermeture de plus en plus systématique des signes et l'emploi d'un module étroit ne laissent guère de place pour l'adjonction d'une ligne complémentaire à but ornemental. L'art de l'inscription ne s'exprime donc plus, à la fin du Moyen Âge, dans la surenchère calligraphique mais au contraire dans le souci de simplifier et d'harmoniser les lignes. Les inscriptions des XIII ${ }^{\mathrm{e}}$ et XIV $^{\mathrm{e}}$ siècles se dépouillent dans leur mise en page, abandonnant peu à peu les cadres richement ornés, les ajouts ornementaux et les compléments calligraphiques. De même que la forme des lettres, la mise en page des inscriptions tend de plus en plus à se rapprocher du modèle livresque et finit par ressembler, dans certains exemples $\mathrm{du} \mathrm{Xv}^{\mathrm{e}}$ siècle, à une "page lapidaire ».

Cette évolution conduira aussi à systématiser la séparation de tous les mots du texte, qu'il soit tracé en majuscules ou en minuscules. La compréhension est facilitée par l'emploi d'une "écriture séparée et normalisée»; l'espace dépourvu de signe avant et après le mot permet d'isoler visuellement chaque terme du texte et matérialise, sur le support épigraphique, la construction logique des expressions, puis des phrases ${ }^{109}$. La séparation des mots devient d'autant plus indispensable à la fin du Moyen Âge que les inscriptions tendent à croître en longueur et en complexité ${ }^{110}$. Le lecteur attend alors une structuration visuelle qui facilite la reconnaissance des signes et la compréhension du message. Dans la petite église de Champniers (Vienne), on trouve une inscription tracée en 1404 pour commémorer la fin des travaux de construction de l'édifice ${ }^{111}$. Le texte est rédigé en langue vernaculaire sur huit lignes, à l'aide de minuscules gothiques régulières. Chaque mot est séparé du précédent par un espace vierge, comme à la cinquième ligne où des espaces séparent le prénom, le nom, le verbe, le complément d'objet direct et le complément de ce dernier ; la structure de la phrase apparaît ainsi visuellement et la compréhension en est

107. CIFM, t. 15, n 103 , p. 100.

108. On rencontre encore par exemple des $A$ à double traverse en 1298 en Normandie (CIFM, t. 22, n $\mathrm{n}^{\mathrm{O}} 29$, p. 68), en 1299 dans le Languedoc (CIFM, t. 11, $\mathrm{n}^{\mathrm{O}} 70, \mathrm{p}$. 86) et à Lyon au milieu du $\mathrm{XIV}^{\mathrm{e}}$ siècle.

109. P. Saenger, «Coupure et séparation... », p. 455 : l'écriture séparée " permet une correspondance presque parfaite entre signes graphiques et unités logiques ".

110. Sur les caractéristiques générales des inscriptions de la fin du Moyen Âge en France, voir R. Favreau, "Les inscriptions du $\mathbf{x v}^{\mathrm{e}}$ siècle en France", dans Epigraphik 2000 : neunte Fachtagung für mittelalterliche und neuzeitliche Epigraphik, Klosterneuburg, 9-12. Oktober 2000, éd. Gertrude Mras et Renate Kohn, Vienne, 2006 (Österreichische Akademie der Wissenschaften, Forschungen zur Geschichte des Mittelalters, 10), p. 131-151.

111. Inscription citée dans Bulletin de la Société des antiquaires de l'Ouest, 1853-1855, p. 307. 
grandement facilitée. L'inscription gravée sur le tombeau de l'écuyer Jean Boilesve, conservée aujourd'hui à la Société des antiquaires de l'Ouest à Poitiers (Vienne) ${ }^{112}$, présente une écriture très proche. La mise en page est toutefois plus sobre, le texte étant gravé directement sur le couvercle, sans cadre ni réglure. Cela n'a pas empêché le lapicide de prendre soin de séparer chaque mot par un espace laissé vierge, de taille importante, surtout dans la première ligne.

Contrairement aux cas qui peuvent encore se rencontrer par exception dans les manuscrits, on ne trouve plus, à compter de la fin du XIII ${ }^{\mathrm{e}}$ siècle, d'inscriptions présentant des exemples de scriptio continua. La lisibilité du texte est au contraire renforcée par l'emploi de plus en plus systématique de la ponctuation. Le nombre de signes en usage n'augmente pas véritablement vers la fin du Moyen Âge, et le ou les point(s) matérialise(nt) souvent dans les inscriptions toutes les pauses, comme dans le manuscrit des XIII $^{\mathrm{e}}-\mathrm{XIV}^{\mathrm{e}}$ siècles ${ }^{113}$. Dans l'épitaphe de Jean Boilesve, un point profondément gravé dans la pierre marque la fin grammaticale et matérielle du texte, après le mot amen; dans le texte de Champniers, c'est une ponctuation par deux points qui joue le même rôle. Dans les deux textes, on ne rencontre pas d'autre trace de ponctuation. Le point joue alors le rôle qu'on lui connaît aujourd'hui. Dans d'autres cas, la valeur grammaticale de la ponctuation reste plus aléatoire. Dans la ferme de la Grande Vacherie près de Poitiers, une inscription en vers français rappelle la construction d'un mur de clôture en $1332^{114}$. Deux points, parfois trois, séparent les mots de façon systématique ou presque. Les mêmes signes sont également gravés au début et à la fin des douze lignes du texte (ill. 14). La ponctuation n'a alors pas valeur de pause ; elle n'aide pas non plus à la prosodie du texte versifié. Elle a valeur de décor, dans le cas présent, mais elle aide surtout à la lisibilité du texte dans une inscription relativement compacte. La hiérarchisation de la ponctuation (pauses fortes et faibles) reste de fait assez rare. Parfois dans les inscriptions métriques, un seul point sépare les mots et deux points séparent les vers, comme dans l'épitaphe de Nicolas de Frécot, inhumé dans l'ancienne abbaye Saint-Florentin de Bonneval (Eure-et-Loir) ${ }^{115}$. Avec l'apparition de la minuscule dans les inscriptions, l'usage de la majuscule au début de la phrase va se généraliser. Le musée de Dreux conserve la deuxième version de la dédicace de la collégiale Saint-Étienne (ill. 15), refaite au $\mathbf{X v}^{\mathrm{e}}$ siècle ${ }^{116}$. Transcrite en minuscules gothiques, elle reprend le contenu de la version du XII ${ }^{\mathrm{e}}$ siècle mais

112. Inscription signalée et lue par Pierre-Amédée Brouillet, Notice des tableaux, dessins, gravures, statues, objets d'art anciens et modernes, curiosités, etc., composant les collections de la ville de Poitiers, Poitiers, 1886, t. II, $\mathrm{n}^{\circ} 304$.

113. J. Vezin, «La ponctuation ... », p. 443-446, p. 443.

114. Inscription présentée pour la première fois dans Bulletin archéologique, t. 3, 1844-1845, p. 120.

115. Un dessin de la dalle a été publié par l'abbé Haye, Dalles tumulaires et pierres tombales du département d'Eure-et-Loir, $3^{\mathrm{e}}$ livraison, Chartres, s. d., $\mathrm{n}^{\circ} 9$ et pl. IX.

116. Dans la salle médiévale du musée de Dreux, on trouve les deux versions de ce texte ; la première est encastrée dans le mur de l'abside reconstituée présentant les fragments des vitraux de Saint-Étienne de Dreux ; la seconde, du $\mathrm{xv}^{\mathrm{e}}$ siècle, est exposée dans une vitrine. 


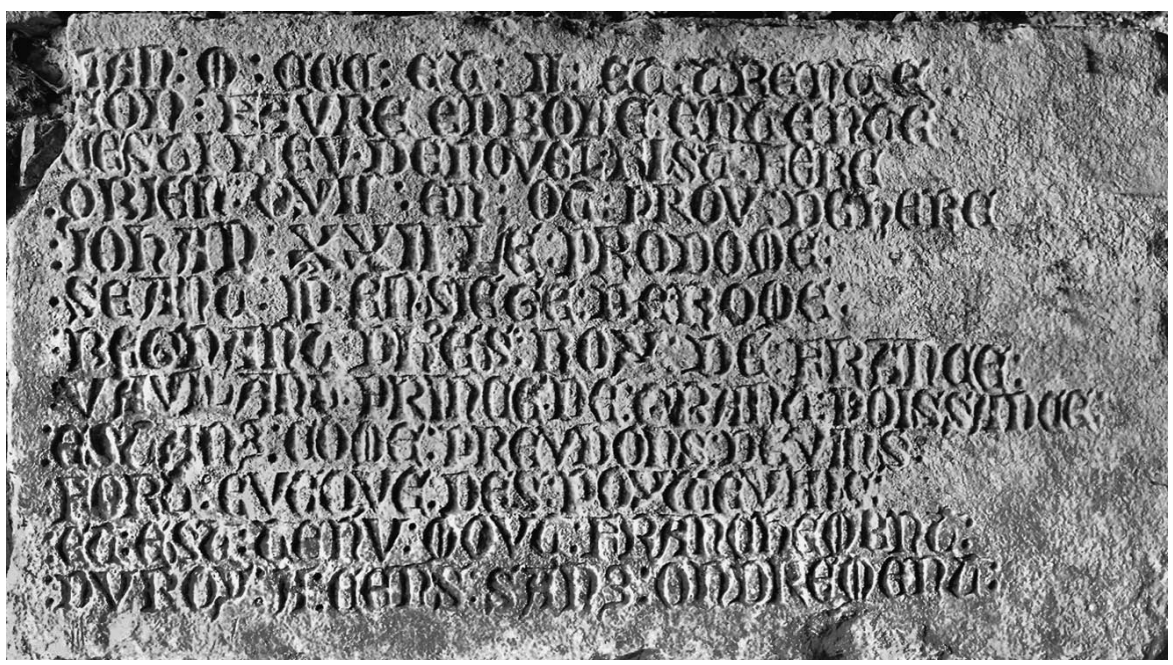

ILL. 14. - Inscription rappelant la construction d'un mur de clôture, 1332. Poitiers (Vienne), ferme au lieu-dit la Grande Vacherie, mur de clôture de la cour. Cliché J. Michaud (CEscm/CIFM).

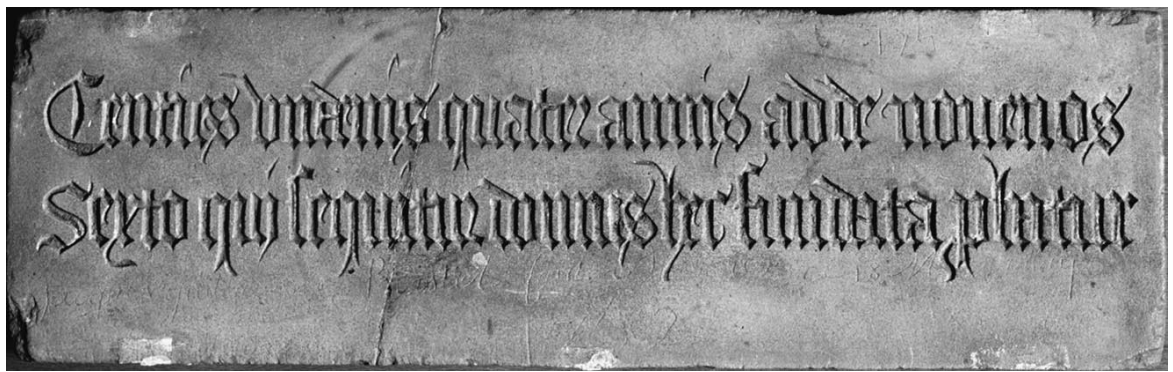

ILL. 15. - Inscription rappelant la construction de la collégiale Saint-Étienne. Dreux (Eure-etLoir), musée des Beaux-Arts, salle médiévale, vitrine. Cliché J.-P. Brouard (СЕsсм/сіғм).

non la ponctuation. De fait, aucun signe n’apparaît sur la pierre et la majuscule au début de chacun des deux vers est le seul signe qui structure le texte.

Le rapprochement entre formes épigraphiques et formes manuscrites de l'écriture à la fin du Moyen Âge repose également sur une ordinatio de plus en plus semblable. Aux $\mathrm{XIII}^{\mathrm{e}}-\mathrm{XV}^{\mathrm{e}}$ siècles, les inscriptions continuent à soigner la mise en page générale du texte mais elles utilisent pour cela des méthodes assez différentes de celles des époques antérieures. Les réglures se font de plus en plus discrètes à la surface de la pierre. Si le texte peut encore s'inscrire entre des traits de préparation, ceux-ci disparaissent souvent de l'inscription finale et ne participent que rarement à son décor. Avec le développement de la minuscule, qui suppose deux ou trois hauteurs de haste, il deviendra de plus en plus 
difficile de tracer des réglures véritablement utiles. L'ordinatio ne perd pas pour autant en qualité. L'équilibre général du texte sur la pierre s'appuie désormais sur une mise en forme plus régulière qui cherche à donner à l'inscription l'apparence d'une " page » lapidaire. Le recours à des caractères de petit module, de proportions allongées, et la multiplication des abréviations donnent une cohérence à la composition et lui confèrent le statut réel de texte, au sens étymologique du terme. Dans les inscriptions, il s'agit d'une nouveauté et l'aspect général devient plus compact, plus dense. Si la capacité à transmettre le message ne diminue pas, cette mise en forme, que l'on rencontre par exemple dans l'une des belles inscriptions de la cour du musée de Chartres ${ }^{117}$, appelle une forme de lecture différente, plus au contact de la pierre. Le cadre qui limite de telles inscriptions est alors rarement orné puisqu'il ne sert plus à convertir le texte en tableau ou en affiche, comme aux époques précédentes, mais en page d'écriture.

La parenté entre écriture épigraphique et écriture des livres se note enfin dans l'adoption progressive par les inscriptions des normes de l'écriture traditionnelle. C'est en particulier le cas des abréviations. Limitées dans le haut Moyen Âge aux nomina sacra, les abréviations dans les textes des XIII ${ }^{\mathrm{e}}$ $\mathrm{XV}^{\mathrm{e}}$ siècles se diversifient. Elles affectent, au cœur de textes de plus en plus longs et de plus en plus riches, des termes nombreux. En revanche, leur forme reste fixe et suit les usages de l'écriture livresque. Le principal changement réside dans la disparition du tilde à renflement médian et, en règle générale, dans une simplification des marqueurs de l'abréviation (tilde droit ou incliné, lettre barrée, etc.). On assiste aussi à la multiplication des lettres inscrites en exposant, notamment pour les dates, ou suscrites.

Les formes livresques de l'ordinatio ne se retrouvent pas, en revanche, dans un type d'inscription dont le développement et la diffusion à la fin du Moyen Âge sont tels que cela affecte statistiquement l'évolution brossée ici à grands traits. Il s'agit du phénomène épigraphique de la plate-tombe, expression qui désigne une dalle de grande dimension recouvrant ou matérialisant au sol la sépulture d'un défunt, la plupart du temps représenté en creux sur la surface. Elle est généralement accompagnée d'une inscription funéraire identifiant le défunt ou commentant sa vie, gravée sur une seule ligne en bordure, autour de la dalle. La dimension et la forme des caractères sont soumises à l'organisation du texte autour de l'image et à la place disponible sur la plate-tombe. La mise en page est tout à fait étrangère ici au modèle livresque, le champ épigraphique ne pouvant pas adopter la forme d'une surface quadrangulaire. Cette condition affecte la forme des lettres (avec des bouleversements dans les proportions, la morphologie et parfois l'ornementation des signes), mais également les contraintes de mise en page et de "mise en texte » du contenu funéraire. Or, entre les années 1250 et 1450, les plates-tombes constituent sans doute plus de

117. Il s'agit de l'épitaphe d'Étienne Charron, mort en 1380. On la trouve aujourd'hui dans la cour du musée des Beaux-Arts de Chartres. Le texte se compose de quatorze lignes réparties à l'intérieur d'un champ épigraphique dont les proportions rappellent celle d'une page de livre. 
$70 \%$ de la documentation épigraphique française. Le rapprochement entre forme épigraphique et forme livresque ne saurait donc s'imposer à l'intégralité des inscriptions de la fin du Moyen Âge. Il n’en représente pas moins une relative homogénéisation des pratiques dans le cadre général de la culture écrite des $\mathrm{XIII}^{\mathrm{e}}-\mathrm{XV}^{\mathrm{e}}$ siècles.

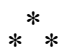

L'étude de l'écriture épigraphique a encore beaucoup de chemin à faire. Elle est tributaire d'une couverture photographique aussi large que possible du territoire. Le développement de l'informatique, avec les facilités d'agrandissement des images, devrait aussi favoriser à l'avenir les études paléographiques.

Les considérations ci-dessus reposent sur une très large moitié sud de la France, mais on ne saurait généraliser celles qui n’ont pas encore été vérifiées sur des exemples de la France du nord et de l'est. Il est indispensable de tenir grand compte du support (pierre, ardoise, métal) et des auteurs de l'inscription (lapicide, orfèvre, peintre). Particulièrement difficile est l'établissement d'une paléographie des inscriptions des peintures murales, dans la mesure où celles-ci ne sont presque jamais datées avec précision. Il est clair qu'on a affaire à des différences régionales, que le lieu de production peut entraîner de nouvelles différences (selon qu'il s'agit d'une paroisse rurale ou d'une grande abbaye, par exemple). Les éléments secondaires peuvent apporter des enseignements utiles, car ils sont moins soumis aux traditions du graveur. L'étude lettre à lettre doit encore trouver ses procédés les plus efficaces : classement par dates? par formes de la lettre? par régions? par supports ? Si la constitution d'une base de données peut réduire la difficulté de méthode préalable, elle ne règle pas la question de la pertinence de l'association avec d'autres éléments constitutifs de l'écriture, réglure ou décor des lettres par exemple. Dans les systèmes d'abréviation aussi, il reste à déceler des évolutions et des variations selon les lieux de production ${ }^{118}$. Le champ d'étude est large et reste encore en grande partie à défricher, mais il apparaît aujourd'hui indispensable d'associer l'analyse paléographique des manuscrits à celle des témoignages épigraphiques pour comprendre pleinement la culture médiévale de l'écrit.

Vincent Debiais, Robert Favreau, Cécile Treffort.

118. Jean Durliat y insiste à juste titre dans « Écritures " écrites " et écritures épigraphiques : le dossier des inscriptions byzantines d'Afrique ", dans Studi medievali, 3e sér., t. 21, 1980, p. 19-46. 
TABLEAUX

\begin{tabular}{|c|c|c|c|c|c|c|}
\hline $\begin{array}{l}1139-1140 \\
\text { Orange }\end{array}$ & \begin{tabular}{|c|}
1140 \\
Bourg-St-Andéol \\
\end{tabular} & \begin{tabular}{|c|}
1142 \\
St-Gilles \\
\end{tabular} & \begin{tabular}{|c|}
1144 \\
Marseille \\
\end{tabular} & $\begin{array}{c}1145 \\
\text { Saint-Quentin } \\
\end{array}$ & $\begin{array}{l}1144 \\
\text { Elne } \\
\end{array}$ & $\begin{array}{c}1148 \\
\text { Vienne } \\
\end{array}$ \\
\hline AAA $\Omega$ & $A \pi$ & $A$ & $A$ & 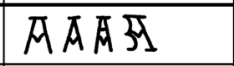 & Aתn & $A s A$ \\
\hline$B$ & $B b$ & & $B$ & B & $B b$ & $B$ \\
\hline$C\{[$ & C & $C[$ & C & C & $C[$ & C \\
\hline DOO & & $D$ & D & 100 & D & $D$ ○ \\
\hline$E \in \in \epsilon$ & $E \epsilon$ & $E$ & $E$ & $E \epsilon$ & $E \in \epsilon$ & $E \epsilon$ \\
\hline$F F$ & $\mathrm{FF}$ & & $F$ & & & $F$ \\
\hline 6 & & G & 60 & 6000 & 6 & G \\
\hline b & $h b$ & $H$ & $H$ & Nhth & $h$ & $\mathrm{Hb}$ \\
\hline \multirow[t]{2}{*}{ I } & 1 & 1 & 1 & 1 & 1 & 1 \\
\hline & & & & & & K \\
\hline L & L & $\mathrm{L}$ & $L$ & L & L & L \\
\hline MMMMI & Mmonon & $M$ & $M$ & $M \Omega$ & $M$ & $M G A C D$ \\
\hline NHNחR & $\mathrm{NN}$ & $N N$ & $N$ & $N \cap \Omega$ & $\mathrm{H}$ & $N$ 周 \\
\hline 0808 & 0 & 0 & 0 & 00 & 08 & 0 \\
\hline$P P$ & $P$ & $P$ & $P$ & P & $P$ & $P$ \\
\hline 0.9 & 9 & & Q & a & 9 & Q9 \\
\hline RRR & $R$ & RR & $R$ & $R R R$ & RP & RRR \\
\hline SSS & $S$ & $S$ & $S$ & $S$ & 5592 & $S$ \\
\hline$\tau \tau$ & $\tau \tau$ & $T \tau$ & $T$ & TTढరठ & $T$ & $T \tau$ \\
\hline$V V V$ & V & V & V & VU & V & VU \\
\hline \multirow[t]{3}{*}{$X$} & & $x$ & $x$ & & $x$ & $x$ \\
\hline & & & & & & \\
\hline & & & & & h & \\
\hline
\end{tabular}

ТАв. 1. - Exemples d'alphabets épigraphiques de la décennie 1140. 


\begin{tabular}{|c|c|c|c|c|}
\hline 597 & $A$ & F & $M$ & $T$ \\
\hline 784 & $A A A$ & E & $M$ & $T$ \\
\hline 830-840 & $A$ & $E$ & $M$ & $T$ \\
\hline v. 976 & $A \wedge$ & $E$ & $M$ & $T$ \\
\hline 1048 & $A A$ & $E \in$ & $M M$ & $T$ \\
\hline 1104 & $A A A_{\text {बा }}$ & $E \in$ & MM G & $\mathrm{T} \sigma$ \\
\hline 1168 & $A A \bar{A}$ & $E E \Theta$ & $M m C$ & Тठ \\
\hline 1230 & $A A D$ & $E \in e$ & $\pi \Omega$ & $\tau$ \\
\hline 1298 & 月 & $\theta$ & 且 & T \\
\hline 1300 & $A B \cap$ & $\theta$ & Q & $\pi$ \\
\hline 1400 & a & $B$ & $\eta 2$ & $t$ \\
\hline
\end{tabular}

TAв. 2. - Exemples de formes de quatre lettres $(A, E, M, T)$ entre le $V I^{e}$ et le XIV siècle. 